\title{
A spatially resolved brain region- and cell type- specific isoform atlas of the postnatal mouse brain
}

Anoushka Joglekar (10 1, Andrey Prjibelski², Ahmed Mahfouz 3,4,5, Paul Collier ${ }^{1}$, Susan Lin (D) 6,7, Anna Katharina Schlusche ${ }^{1}$, Jordan Marrocco ${ }^{8}{ }^{8}$, Stephen R. Williams ${ }^{9}$, Bettina Haase ${ }^{10}$, Ashley Hayes ${ }^{9}$, Jennifer G. Chew ${ }^{9}$, Neil I. Weisenfeld ${ }^{9}$, Man Ying Wong ${ }^{11}$, Alexander N. Stein ${ }^{12}$, Simon A. Hardwick ${ }^{1,13}$, Toby Hunt ${ }^{14}$, Qi Wang ${ }^{15}$, Christoph Dieterich ${ }^{15}$, Zachary Bent ${ }^{9}$, Olivier Fedrigo ${ }^{10}$, Steven A. Sloan ${ }^{16}$, Davide Risso ${ }^{17}$, Erich D. Jarvis ${ }^{10,18}$, Paul Flicek (1) ${ }^{14}$, Wenjie Luo (D) ${ }^{11}$, Geoffrey S. Pitt (1) 6,7, Adam Frankish ${ }^{14}$, August B. Smit ${ }^{19}$, M. Elizabeth Ross (i) ${ }^{1} \&$ Hagen U. Tilgner (i] ${ }^{1 凶}$

Splicing varies across brain regions, but the single-cell resolution of regional variation is unclear. We present a single-cell investigation of differential isoform expression (DIE) between brain regions using single-cell long-read sequencing in mouse hippocampus and prefrontal cortex in 45 cell types at postnatal day 7 (www.isoformAtlas.com). Isoform tests for DIE show better performance than exon tests. We detect hundreds of DIE events traceable to cell types, often corresponding to functionally distinct protein isoforms. Mostly, one cell type is responsible for brain-region specific DIE. However, for fewer genes, multiple cell types influence DIE. Thus, regional identity can, although rarely, override cell-type specificity. Cell types indigenous to one anatomic structure display distinctive DIE, e.g. the choroid plexus epithelium manifests distinct transcription-start-site usage. Spatial transcriptomics and long-read sequencing yield a spatially resolved splicing map. Our methods quantify isoform expression with cell-type and spatial resolution and it contributes to further our understanding of how the brain integrates molecular and cellular complexity.

\footnotetext{
${ }^{1}$ Brain and Mind Research Institute and Center for Neurogenetics, Weill Cornell Medicine, New York, NY, USA. ${ }^{2}$ Center for Algorithmic Biotechnology, Institute of Translational Biomedicine, St. Petersburg State University, St Petersburg, Russia. ${ }^{3}$ Department of Human Genetics, Leiden University Medical Center, Leiden 2333 ZC, The Netherlands. ${ }^{4}$ Leiden Computational Biology Center, Leiden University Medical Center, Leiden 2333 ZC, The Netherlands. ${ }^{5}$ Delft Bioinformatics Lab, Delft University of Technology, Delft 2628 XE, The Netherlands. ${ }^{6}$ Graduate Program in Neuroscience, Weill Cornell Medical College, 1300 York Avenue, New York, NY 10065, USA. ${ }^{7}$ Cardiovascular Research Institute, Weill Cornell Medicine, New York, NY, USA. ${ }^{8}$ Harold and Margaret Milliken Hatch Laboratory of Neuroendocrinology, The Rockefeller University, New York, NY, USA. ${ }^{9} 10 x$ Genomics, Pleasanton, CA, USA. ${ }^{10}$ The Vertebrate Genomes Lab, The Rockefeller University, New York, NY, USA. ${ }^{11}$ Brain and Mind Research Institute and Appel Alzheimer's Research Institute, Weill Cornell Medicine, New York, NY, USA. ${ }^{12}$ School of General Studies, Columbia University, New York, NY, USA. ${ }^{13}$ Genomics and Epigenetics Division, Garvan Institute of Medical Research, Sydney, NSW, Australia. ${ }^{14}$ European Molecular Biology Laboratory, European Bioinformatics Institute, Hinxton, UK. ${ }^{15}$ Section of Bioinformatics and Systems Cardiology, University Hospital, 96120 Heidelberg, Germany. ${ }^{16}$ Department of Human Genetics, Emory University School of Medicine, Atlanta, GA, USA. ${ }^{17}$ Department of Statistical Sciences, University of Padova, Padova, Italy. ${ }^{18}$ Howard Hughes Medical Institute, Chevy Chase, MD, USA. ${ }^{19}$ Department of Molecular and Cellular Neurobiology, Center for Neurogenomics and Cognitive Research, Amsterdam Neuroscience, VU University, Amsterdam, The Netherlands. ${ }_{\text {email: hut2006@med.cornell.edu }}$
} 
A lternative splicing (AS) affects almost all spliced genes in mammals ${ }^{1,2}$, vastly expands the proteome ${ }^{3}$ and increases functional diversity of cell types ${ }^{4}$. Alternative transcription start sites (TSS) and poly-adenylation (polyA) sites further expand the alternative isoform landscape, regulating development, differentiation, and disease $\mathrm{e}^{5-9}$. These RNA variables often depend on each other ${ }^{10-13}$, and how their combined status impacts individual molecules can only be assessed using longread sequencing ${ }^{11,12,14-17}$, which sequences transcripts in single reads with no assembly required, thereby reducing alternative transcript assembly errors and enabling accurate isoform quantification.

Brain AS is especially diverse ${ }^{18}$ and brain-region specific expression patterns of splicing factors ${ }^{19}$ and other RNAbinding proteins ${ }^{20}$ drive brain-region-specific splicing. Examples include diseases implicated by genes such as MAPT, Bin1, and neurexins ${ }^{16,17,21}$. Brain-region-specific isoform expression can either originate from molecular regulation in one or multiple cell types, or can arise purely from gene-expression or celltype abundance differences without splicing regulation. These distinct models are especially important during postnatal development. For instance, in hippocampus (HIPP) and prefrontal cortex (PFC), multiple cell types undergo differentiation, which is influenced by development-specific splicing ${ }^{1,22-}$ 25 distinct from that of mature cell types. However, no celltype-specific isoform investigation across brain regions exists to-date, owing to limitations in technology, throughput, and testing methods. HIPP and PFC are highly specialized regions of the telencephalon, and their circuitry is heavily implicated in movement control, cognition, learning, and memory formation. Disorders involving HIPP and PFC manifest in cognitive deficits, and understanding changes occurring at crucial developmental timepoints of these structures is important for case-control studies. Here, we employ single-cell isoform RNA sequencing (ScISOrSeq) ${ }^{26}$ with increased throughput in HIPP and PFC at mouse postnatal day 7 (P7) to test and define celltype-specific contributions to brain-region-specific splicing. Furthermore, we devised a spatial isoform expression method, which provides a spatial exon expression map (see www. isoformAtlas.com) in addition to the existing spatial geneexpression map of the Allen developing brain atlas.

\section{Results}

Short read clustering of P7 hippocampus and prefrontal cortex tissue assigns precursors to known adult cell-types. Our ScISOrSeq approach used barcoded single cells followed by both short and long-read analyses to reveal splice variants specific to cell types (Fig. 1a). We identified cell types first using single-cell $3^{\prime}$-end sequencing. Short-read clustering across two hippocampal replicates revealed no need for integration anchors ${ }^{27}$ to correct for batch effects (Fig. S1a). Characteristic markers ${ }^{28,29}$ for 24 clusters in HIPP (Fig. S1b) identified eight glial types, including two astrocyte, three oligodendrocyte, a radial glia like (RGLs), ciliated ependymal, and secretory choroid plexus epithelial (CPE) cell clusters. Furthermore, we observed six vascular and immune populations including vascular endothelial cells, microglia, and macrophages (Fig. 1b). RNA velocity analysis revealed neuronal lineages in various differentiation stages (Fig. S1c, d): a neuronal intermediate progenitor cell (NIPC) population; three dentate gyrus granule neuroblast clusters (DG-GranuleNB); and three clusters each of excitatory (EN) and inhibitory neurons (IN). Alignment of our P7 data with published P30 hippocampal data ${ }^{30}$ revealed subtype identities (CA3, CA1, Subiculum) for three excitatory neuron clusters and medial ganglionic eminence (MGE) and non-MGE-derived interneurons ${ }^{31-33}(P V+, S s t+$,
Lamp5 +, Vip +) in one cluster (IN1), distinct from Cajal-Retzius (IN3) cells (Fig. 1b, Supplementary Fig. 1e-g).

Similar analysis in PFC revealed seven glial clusters including astrocytes, oligodendrocytes, six populations of vascular and immune cells, and seven neuronal types ${ }^{34,35}$ with confirmation of intermediate states from RNA velocity (Fig. 1c, Fig. S2a-d). Alignment with public P30 cortex data ${ }^{30}$ further subdivided neuronal clusters into known cortical excitatory and interneuron classes (Fig. S2e-g). In contrast to HIPP, the MGE $(P V+, S s t+)$ and non-MGE interneurons (Vip +, Lamp5 +) in the PFC were better separated into two clusters (IN1, IN2), while Cajal-Retzius cells again clustered separately (IN3). We identified excitatory neurons corresponding to different cortical layers which are not well-differentiated at P7. P4 ISH images (Image credit: Allen Institute) alongside gene expression projected onto the UMAP plots further validated our cell-type identification for both regions (Fig. 1d).

A gene-wise test to determine differential isoform expression (DIE). We next conducted long-read sequencing on our singlecell full-length HIPP and PFC cDNA (Supplementary Table 1), and deconvolved reads for each cell type using single-cell barcodes (Fig. 1a) for two independent replicates (Fig. S3, SS3). Differential exon usage between two conditions has been successfully assessed using a $2 \times 2$ contingency table per exon ${ }^{2}$. Using this method on our long-read data from HIPP and PFC yielded 31 genes $(1.45 \%, n=2132)$ exhibiting differential exon usage after Benjamini-Yekutieli (BY) correction ${ }^{36}$ for dependent tests (Fig. S5a). Given this harsh correction, we devised a more sensitive gene-level test that considers TSS and polyA-sites in addition to exon connectivity. In this test, we count isoforms per gene in both conditions, leading to a $\mathrm{n} \times 2$ table. This yields fewer and independent tests, allowing for the Benjamini-Hochberg (BH) correction $^{36}$ and reducing false negatives. In each brain region, we define "percent isoform" (П) as an isoform's relative abundance among its gene's transcripts. Similarly to requiring a $\Delta \Psi \geq 0.1$ for short reads ${ }^{2}$, we require $\mathrm{FDR}<=0.05$ and $\Delta \Pi \geq 0.1$. This $\Delta \Pi \geq$ 0.1 can be contributed collectively by at most two isoforms, provided their isoform $\Delta \Pi$ point in the same direction, to consider a gene exhibiting differential isoform expression (DIE, Fig. 2a).

In contrast to the 31 significant genes derived by the exon-based test, 395 genes (FDR $<=0.05, \Delta \Pi \geq 0.1 ; 9.06 \% ; n=3958$ ) exhibited DIE when comparing HIPP and PFC isoforms using the gene-level test (Fig. 2b). The multiple testing correction factor influenced the significant gene number. For example, the $\mathrm{H} 13$ gene (Fig. S5b) had a $p$-value of $1.7 \times 10^{-4}$ (uncorrected) and $2.7 \times 10^{-3}$ (corrected) by isoform tests, with a Benjamini-Hochberg correction factor of 15.6. The same gene's alternative exon had a $p$-value of $1.3 \times 10^{-4}$ (uncorrected) and 0.057 (corrected, non-significant) by exon tests with a Bejamini-Yekutieli correction factor of 431.3. Thus, genewise isoform testing is more sensitive (Fig. 2c, d). Concordantly, the maximum $\Delta \Psi$ for genes with DIE is higher than genes without DIE ( $p \leq 0.015$, Wilcoxon-rank-sum test) (Fig. 2e).

Since our gene-level test considers exon connectivity, we can identify the exact isoforms contributing to DIE. Among the top two contributing isoforms for each of the 395 genes exhibiting regional DIE, we identified 76 high-confidence novel isoforms (Methods). Manual validation using GENCODE criteria confirmed all 76. Functionally, 40 (52.6\%) are coding transcripts, 24 (31.6\%) show nonsense mediated decay (NMD), 11 (14.5\%) show intron retention, and one isoform is of a long noncoding gene (Meg3) (Fig. 2f). Such non-coding and NMD transcripts indicate region-specific regulation ${ }^{1}$. To pinpoint the source of isoform differences in the 395 genes, we tested TSS and polyA-site 

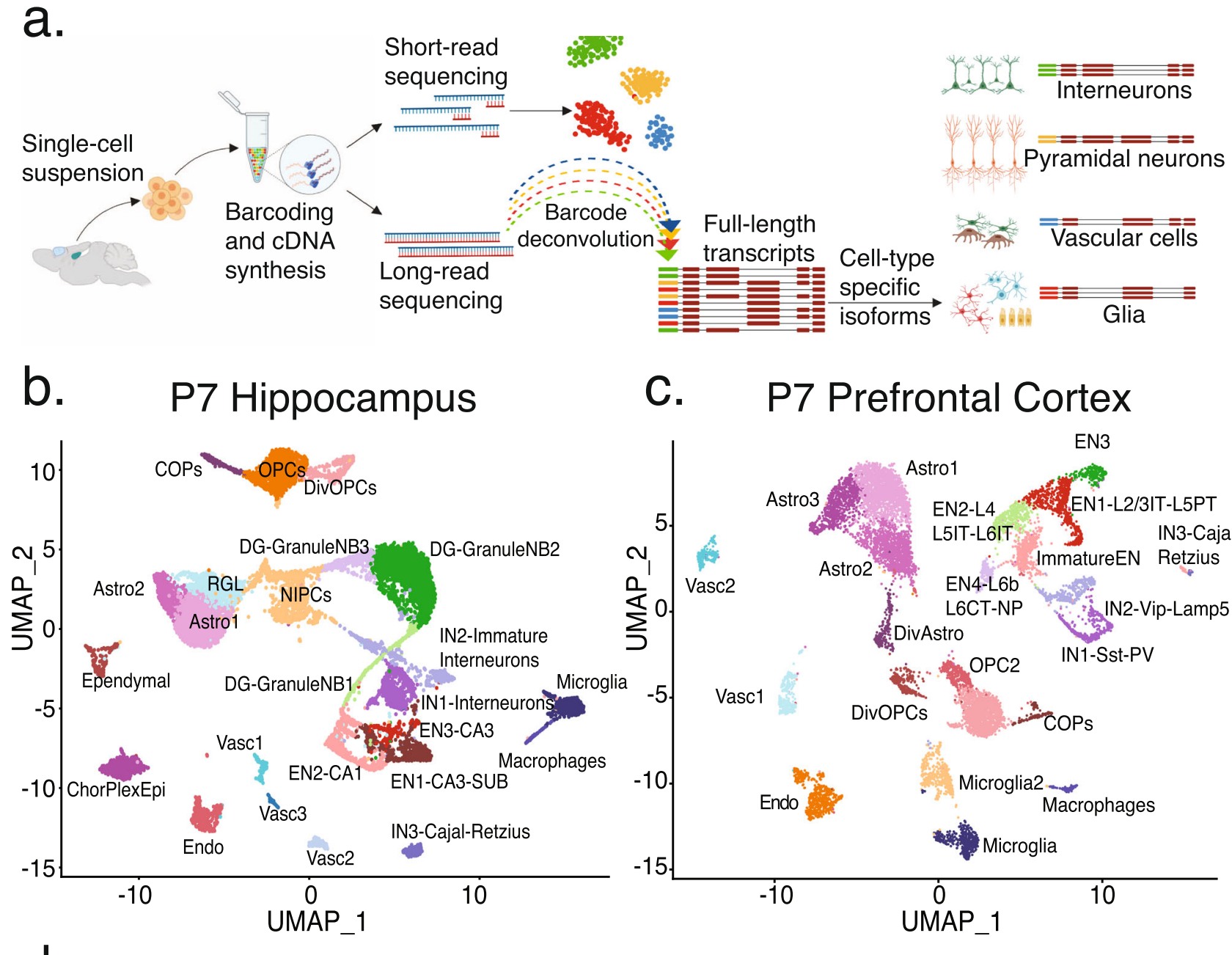

C. P7 Prefrontal Cortex
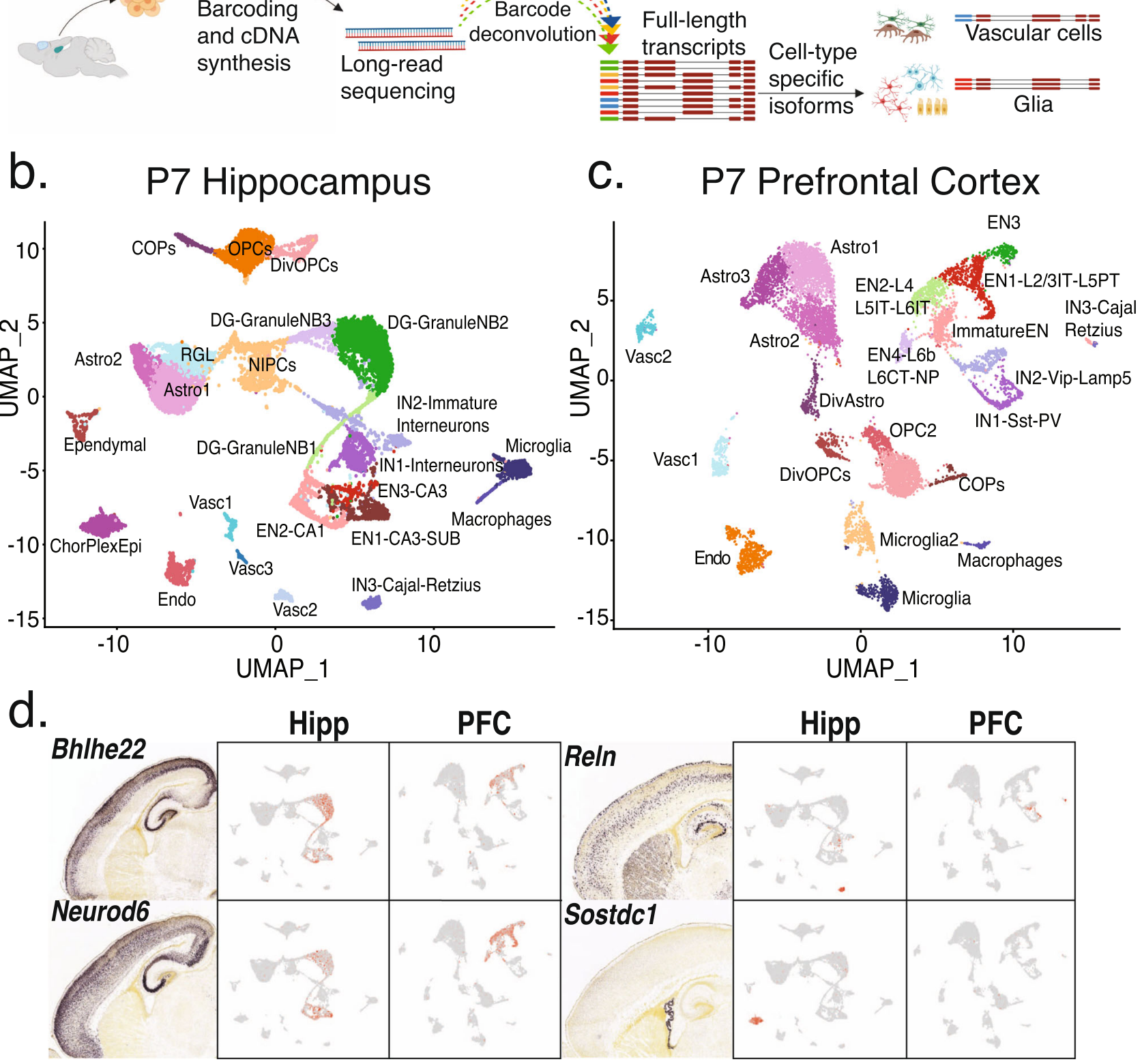

Fig. 1 Short read clustering of P7 hippocampus and prefrontal cortex tissue recovers precursors to known adult cell-types. a Schematic of the ScISOrSeq workflow (created with BioRender.com). b UMAP of P7 hippocampus (HIPP) data. Cell-types identified by marker genes, RNA velocity analysis, and alignment to published data shown in S1. c UMAP of P7 prefrontal cortex (PFC) data. Cell-types identified by marker genes, RNA velocity analysis, and alignment to published data shown in S2. $\mathbf{d}$ In situ hybridization (ISH) images from Allen developing mouse brain atlas for marker genes and corresponding projections on UMAP plots from HIPP and PFC.

abundance per gene across brain regions, which follow the same statistical framework as the isoform tests. 141 (of 395) genes exhibited differential TSS or polyA-site usage (Fig. 2g). By extension, the remaining 254 genes are explained by splice-site usage differences.

Many genes with DIE have $\Delta \Pi \leq 0.5$; however, we also identified drastic switches $(\Delta \Pi \geq 0.5)$ : Nsfllc encodes the Nsfl1 cofactor $\mathrm{p} 47$, which regulates tubular ER formation, influences neuronal dendritic spine formation, and dendritic arborization $^{37,38}$. A 6 nt microexon is preferentially included in HIPP across neuronal and glial cell types but is absent in the same PFC cell types (Fig. 2h). The synaptic gene Nsmf is involved in the cAMP pathway ${ }^{39,40}$ and through nuclear translocation of its protein, in memory formation 39,40 . In our data, the major HIPP isoform is absent in PFC, while the second HIPP isoform represents the majority of that gene's PFC expression. The isoforms differ by a 69nt exon with a nuclear localization signal and one of two synaptic targeting elements. 

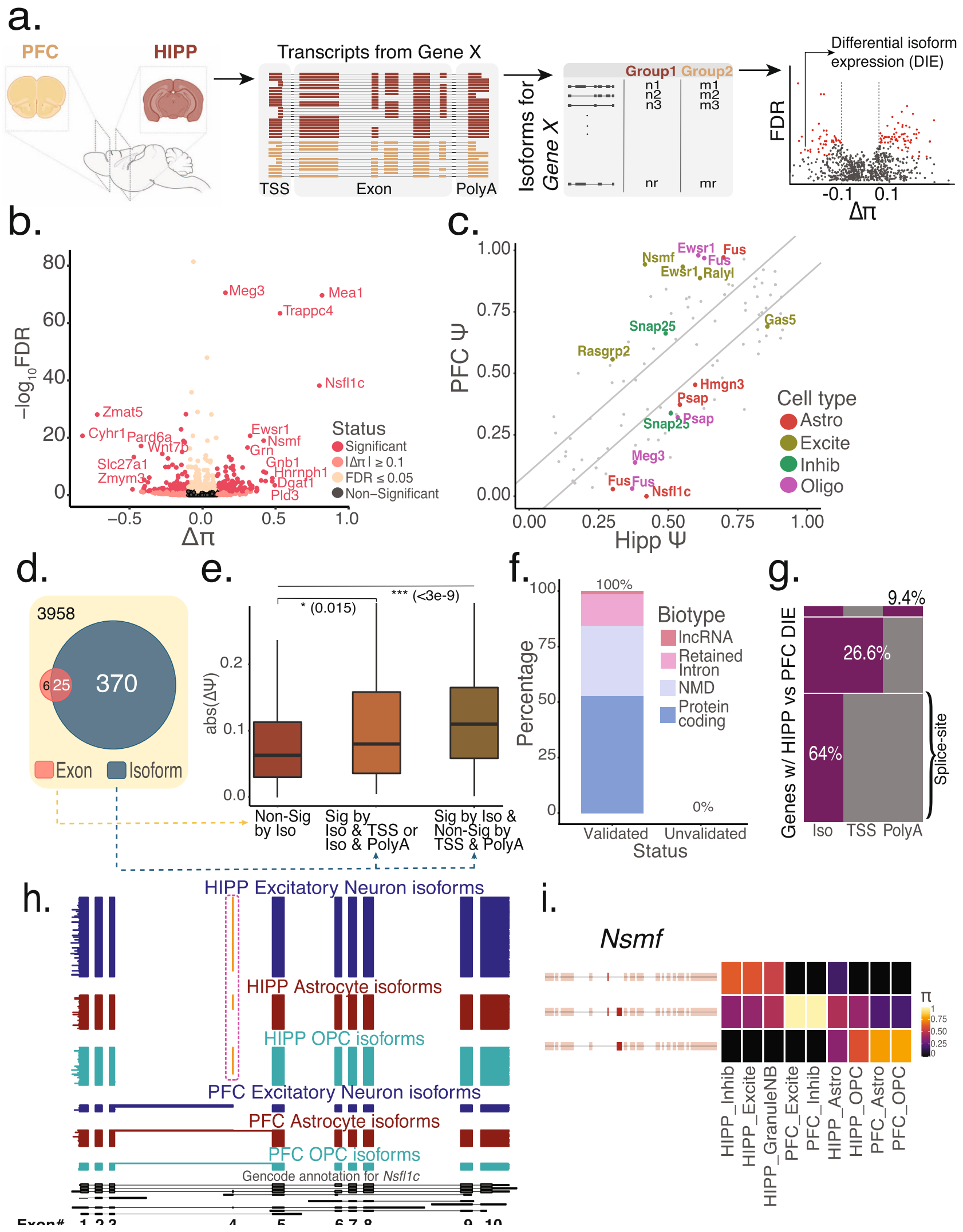

Hence, this exon may affect the synapse to nucleus signaling that the protein is involved in. A third Nsmf isoform with the $69 \mathrm{nt}$ exon but lacking a $6 \mathrm{nt}$ micro-exon is favored in PFC over HIPP but completely missing from neuronal cells, highlighting the regulatory role of micro-exons in neuronal function 1,41 (Fig. 2i).
Differential isoform expression across brain regions is governed predominantly by one specific cell type. Gene expression transcript similarities among clusters defined a cell-type hierarchy, first separated by neurons and non-neurons, and then by other cell types (Fig. S6). Since inhibitory neuron types are transcriptionally more similar to each other than to excitatory 
Fig. 2 A gene-wise test for differential isoform expression (DIE) is more sensitive than an exon-wise test at detecting splicing changes. a Schematic of the scisorseqR approach-Barcode deconvolution, filtering, pairwise comparison, and reporting of significant results based on FDR and $\Delta \Pi$ cutoffs (created with BioRender.com). $\mathbf{b}$ Volcano plot of bulk HIPP vs. bulk PFC differential abundance analysis, with the effect size $(\Delta \Pi)$ on the $X$-axis. $P$-values derived from a $\chi^{2}$ test and corrected for multiple testing using the Benjamini-Hochberg correction are plotted on the $Y$-axis. Points are colored according to the levels of significance based on FDR and $\Delta \Pi$ value. Genes considered significant (pink) when FDR $\leq 0.05$ and $|\Delta \Pi| \geq 0.1$. c Scatter plot showing the $\Delta \Psi$ of all exons for genes that show significant DIE between HIPP and PFC. Gray points represent non-significant exons. Points are colored according to the celltype in which an exon is considered significant by a BY corrected $p$-value and a $\Delta \Pi \geq 0.1$. Diagonal lines indicate cutoff of $0.1 \Delta \Psi$. $\mathbf{d} \vee$ enn diagram showing the overlap of genes significant by DIE (BH correction) with genes significant by exon tests (BY correction). e Boxplot showing the maximum absolute value of $\Delta \Psi$ per gene in three different categories: genes that are not significant by DIE tests $(n=1395)$, genes that are significant by DIE tests and also exhibit differential TSS or polyA-site usage $(n=38)$, and genes that are significant by DIE and do not exhibit differential TSS or polyA-site usage $(n=128)$. $P$-values were calculated using a two-sided Wilcoxon rank sum test. Data are represented as boxplots where the middle line is the median, the lower and upper hinges correspond to the first and third quartiles, the upper whisker extends from the hinge to the largest value no further than $1.5 \times I Q R$ from the hinge (where IQR is the inter-quartile range) and the lower whisker extends from the hinge to the smallest value at most $1.5 \times$ IQR of the hinge. Please see function geom_boxplot in R (ggplot2). $\mathbf{f}$ Percentage of novel transcripts by scisorseqR that were manually validated as being novel by Gencode team, and breakdown of predicted function. $\mathbf{g}$ Heatmap of significant DIE genes ( $n=395$, BH correction) according to entire isoform between bulk HIPP and bulk PFC that also exhibit differential usage of transcription start site (TSS) and polyA-site (PolyA). Each row is a single gene. Gray represents genes that are nonsignificant by category (Iso/TSS/PolyA) whereas purple represents significant by category. $\mathbf{h}$ Isoform expression of Nsfl1c gene. Each horizontal line in the plot represents a single transcript colored according to the cell-type it is represented in. Therefore, blocks represent exons and whitespace represents intronic space (not drawn to scale). Orange exon represents alternative inclusion. $\mathbf{i}$ Isoform expression for the Nsmf gene. Each row represents an isoform colored by $\Pi$ and each column represents a cell-type in HIPP or PFC.

types $^{34,35}$, we grouped finer inhibitory neuron subtypes (IN1, IN2, IN3) into a composite inhibitory neuron (IN) category, and excitatory neuron subtypes into an excitatory neuron (EN) category. We hypothesized three alternative models that could underlie differential isoform expression between brain regions: 1) multiple or all cell types change splice variants ('Both-Cell-TypesModel'); 2) a single cell type changes splice variants ('Single-CellType-Model'); or 3) changes in expression or cell-type abundance without any change in splice variants ('No-Cell-Type-Model') (Fig. 3a).

We analyzed neuronal and non-neuronal reads separately and cross-referenced the results of the 395 genes with DIE. 26 genes $(6.6 \%, \mathrm{FDR}<=0.05)$ had DIE in neurons and non-neurons under the Both-Cell-Types-Model, 151 (38.2\%) in neurons only and $81(20.5 \%)$ in non-neurons only under a Single-Cell-TypeModel, and 137 (34.7\%) that were either too low in expression for testing (Methods) in both neuronal and non-neuronal cells or followed the No-Cell-Type-Model (Fig. 3b). To distinguish these two explanations for the 137 genes, we considered genes with $\Delta \Pi \geq 0.1$ irrespective of $p$-values for the 395 genes. Specifically, we calculated the ratio of $\Delta \Pi$ in a finer subtype to the $\Delta \Pi$ in the composite cell type. After dividing all cells (composite) into neurons and non-neurons (finer level), 75\% $\left( \pm 2.3, \mathrm{SE}_{\mathrm{p}}\right)$ of bulk DIE events were traced to only neurons or only non-neurons (Single-Cell-Type-Model, green) and $24.4 \%$ to both (Both-CellTypes-Model, light purple, Fig. 3ci). A single gene followed the No-Cell-Type-Model (0.3\%, dark purple). Similarly, when dividing neurons into excitatory and inhibitory subtypes, we found $78.8 \%( \pm 2.97)$ for the Single-Cell-Type-Model, $19.58 \%$ for BothCell-Types-Model, and 1\% for No-Cell-Type-Model (Fig. 3cii). When separating composite non-neuronal cells into glia and vascular + immune cells, and again when separating glia into astrocytes and oligodendrocytes, and then the vascular + immune cluster into vascular and immune cells, we observe similar trends. The Single-Cell-Type-Model was more prevalent than the BothCell-Types and No-Cell-Type Models (Fig. 3c). These observations were replicated in replicate 2 , despite differences in cell-type proportions between replicates (Fig. S7a-c). In summary, the NoCell-Type-Model is rare, representing $0.3-3.28 \%$ for the different cell group divisions. Extending this observation to the 137 genes above (Fig. 3b), one gene ( $0.3 \%$ of 395 ) likely represents the NoCell-Type-Model whereas the other 136 can be attributed to low read depth.
An example of the dominant Single-Cell-Type-Model is Hexosaminidase A (Hexa), which is implicated in Tay-Sach's disease $^{42}$. In addition to a single annotated isoform, PFC excitatory neurons show significantly diminished inclusion of an internal exon (from $81 \%$ inclusion in HIPP to $22 \%$ ), thus expressing a novel isoform. Other cell types show no difference between HIPP and PFC. Manual validation classified this novel isoform as NMD, indicating brain-region and cell-type-specific NMD (Fig. 3d).

Brain regions can override cell-type specificity for a subset of genes, possibly through microenvironmental influence. Despite the prevalence of the Single-Cell-Type-Model, the Both-Cell-TypesModel is still common. To avoid circular reasoning, we considered all genes sufficiently expressed (Methods) in neurons and nonneurons in both brain regions. Concurrent regional DIE differences $(\Delta \Pi \geq 0.1)$ in both neurons and non-neurons occur more often than expected by chance $\left(\frac{\text { observed }}{\text { expected }}=\frac{23.9 \%}{15.6 \%}=1.5, p \leq 2.2 e^{-16}\right.$, Fisher's two-sided exact test). This trend is also conserved in excitatory and inhibitory neurons $\left(p \leq 2.2 e^{-16}\right)$ as well as in glia and vascular + immune cells (Fig. 3e). Not only do we see concurrence in Rep1, but we find that the observation is conserved in all investigated levels across both replicates considered together (Fig. S7d). Two nonmutually exclusive models may underlie this observation-microenvironment and cell origin. Firstly, HIPP and PFC interneurons originate in the ganglionic eminences ${ }^{31,33}$, while excitatory neurons do not. Thus, splicing similarities between HIPP EN and IN that are different from PFC EN and IN might be imposed by the regional microenvironment. Secondly, considering neurons and glia, their common descendance from radial glial stem cells may underlie cases of brain-region-specific regulation that overrides cell-type specificity.

Cell types endogenous to one brain region have distinct splicing signatures. We traced the contribution of individual cell types to bulk DIE. Region-specific DIE was clearly traceable $\left(\frac{\Delta \pi_{\text {cell-type }}}{\Delta \pi_{\text {Bulk }}} \geq 0.9\right)$ in $73.4 \%(n=395)$ of the cases, while $10.4 \%$ had $\frac{\Delta \pi_{\text {cell-type }}}{\Delta \pi_{\text {Bulk }}} \leq 0.9$ in all cell types (Fig. S7e, f). The remaining $16.2 \%$ had low read counts in all cell types. Some genes truly have a regional component i.e., concurrent changes across multiple cell types (Fig. S7f). The low depth and $\Delta \Pi$ ratio could arise from cell 

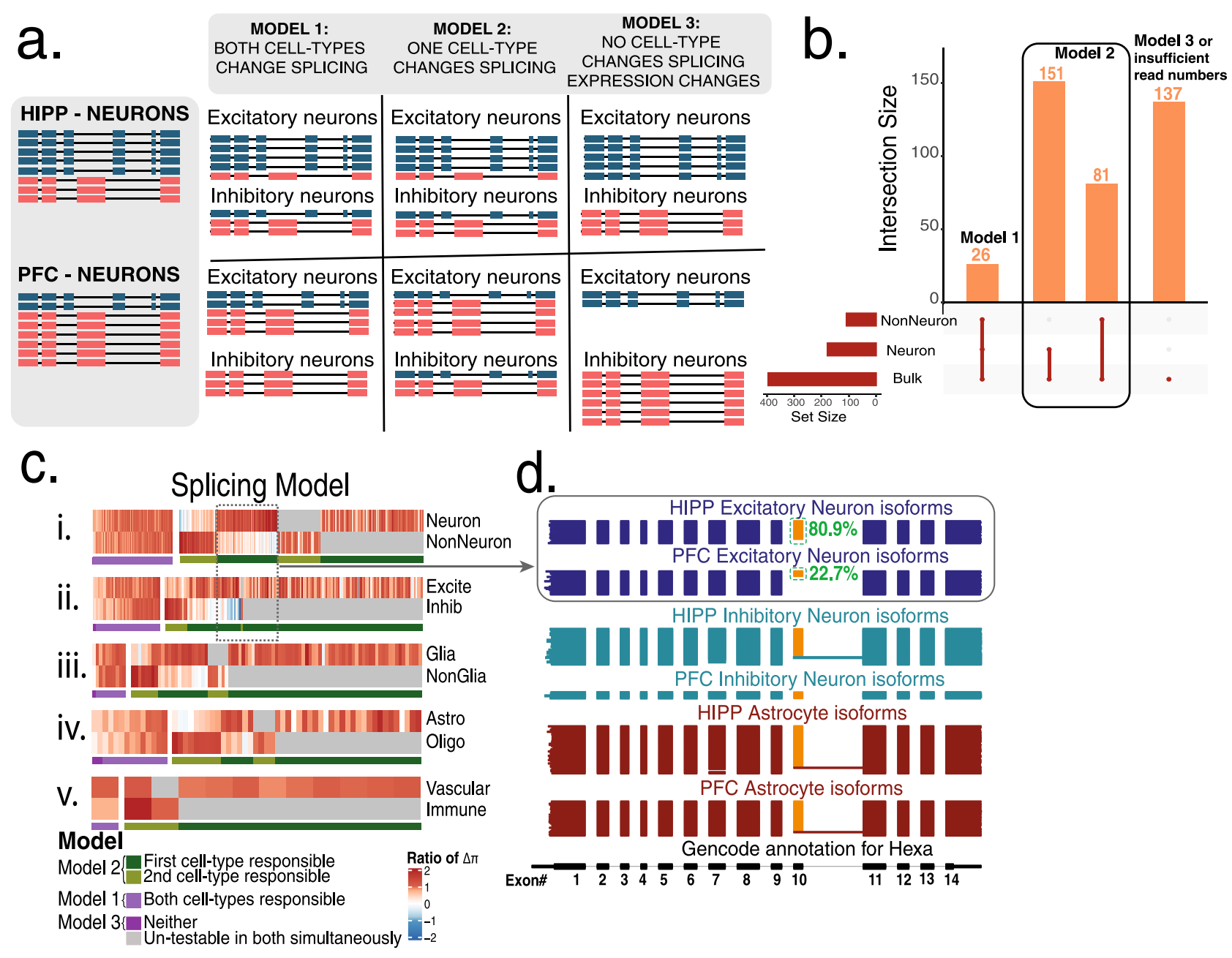

d.

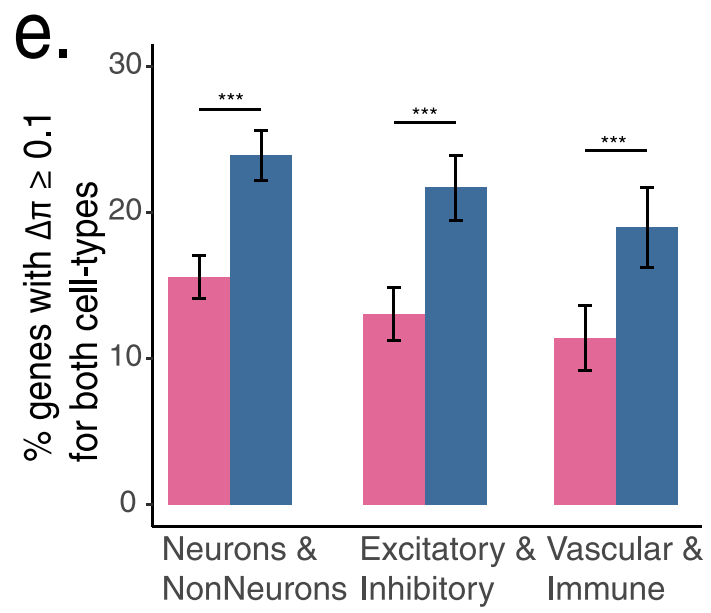

\section{Variable Expected Observed}

Fig. 3 Three models of alternative splicing followed by differentially spliced genes across brain regions. a Schematic of three models that explain splicing changes between any two categories (e.g., HIPP neurons versus PFC neurons). b Upset plot of DIE genes in bulk, neuron, and non-neuron. c Five gene $\times$ celltype heatmaps clustered by the ratio of $\Delta \Pi$ of an individual cell-subtype to a parent cell-type i.e., value for Neuron $=\Delta \Pi_{\text {Neuron }} / \Delta \Pi_{B u l k}$. Each vertical line indicates the ratio of $\Delta \Pi$ for a single gene. Gray lines indicate lack of sufficient depth or lack of expression. Clusters of genes are colored by whether both cell-types show similar relative $\Delta \Pi$ to the parent (purple, Model I, Model III) or whether one cell-type explains most of the splicing changes (Model II). d Hexa gene representing an example of Model II. Each horizontal line in the plot represents a single transcript colored according to the cell-type it is represented in. Therefore, blocks represent exons and whitespace represents intronic space (not drawn to scale). Orange exon represents alternative inclusion. e Barplots indicating percent of genes with $|\Delta \Pi| \geq 0.1$ for two concurrently assessed cell-types. Pink bar indicates expected levels assuming independence, while blue bars represent observed levels $(n=2351,1320,785)$. Error bars indicate $95 \%$ confidence interval of proportion. $P$-values calculated using Fisher's two-sided exact test. 
types only present in HIPP but not PFC. Indeed, reads originating from granule NB, RGL, ependymal, and $\mathrm{CPE}$ clusters have a higher ratio in the $26.4 \%$ of genes for which DIE was not explained (gray) than in the genes where DIE was explained (yellow) (Fig. S7g). For example, for the Fxyd1 gene, CPE cells in HIPP had a different splicing signature from astrocytes in HIPP and PFC, leading to regional DIE (Fig. S7h). These observations warrant further exploration of each cell type's splicing signatures.

Choroid plexus epithelial cells (CPEs) generate distinct isoforms predominantly through alternative TSS. We performed DIE tests in pairwise comparisons of HIPP cell types. DIE was most frequent for neuron vs. non-neuron comparisons in HIPP (Fig. 4a), and this was confirmed in PFC (Fig. S8). High percentages were also seen in some comparisons between nonneuronal cell types. Interestingly, comparisons between nonneuronal cell types showed higher DIE than those observed within neurons (Fig. 4b). Importantly, non-neuronal comparisons involving CPEs clearly had the highest DIE fractions. CPEs are cerebrospinal fluid secreting ependymal cells in cerebral ventricles, and alternative splicing in CPEs relates to disease ${ }^{9,43}$ (Fig. 4c). Surprisingly, TSS choice (compared to exons and polyAsites) largely explained the isoform regulation of CPE cells (Fig. 4d). Furthermore, CPE-associated transcripts strongly favored an upstream TSS compared to the non-CPE transcripts (70/93 genes, Bernoulli $p=3 \times 10^{-7}$ ). This can allow for CPEspecific post-transcriptional modifications ${ }^{44}$, translation initiation, and transcription factor control of gene expression (Fig. 4e).

Single-cell basis of DIE between cell types. When DIE is observed between two cell types, two competing hypotheses can explain this phenomenon ${ }^{4}$. Either all cells of each cell-type behave uniformly and reflect the differences in isoform expression between the two cell types, or individual cells of one or both cell types could show variability in isoform expression. Neuronatin (Nnat) is an important developmental gene expressing a neuronspecific isoform. In Nnat, DIE between ependymal cells and excitatory neurons is represented by the vast majority of individual cells. However, the case of DIE between excitatory neurons and granule neuroblasts is different: some granule neuroblasts behave like excitatory neurons, while others behave like nonneurons. This may be due to different sub-populations of granule neuroblasts (Fig. 4f).

Clustering on long-read data recapitulates short-read cell-type assignments. We clustered hippocampal cells using their isoform expression similarities. Compared to $3^{\prime}$ seq clustering, glial, immune, and vascular clusters were similarly defined (Figs. S9a, b and 4a). Jaccard similarity index analysis between short-read and long-read clusters showed high concordance for broad-level classification (Fig. S9c). Additionally, isoforms of some genes are better resolved with long reads than with than $3^{\prime}$-expression short reads, including for distinguishing neurons and non-neurons (e.g., $\mathrm{Pkm}, \mathrm{Clta}, \mathrm{H} 3 \mathrm{f} 3 \mathrm{~b}$ ), or mature and immature neurons (e.g., Cdc42, Srsf3, Thra, Fig. S9d). However, despite striking differences in isoform expression within neuronal subtypes, differences between isoform-derived clusters and short-read derived clusters remained. Long-read clustering successfully separated CA1 from CA3 neurons (i.e., short-read EN1 vs EN2) but did not separate all cells of the more immature IN2 cluster from mature granule neuroblasts i.e., GranuleNB-2 (Fig. S9e, f). Such differences could be explained by cell subtype specificity in isoforms only, or reduced sequencing depth of isoforms.
Relative isoform expression differences during development reflect dynamic changes in function. Using Slingshot ${ }^{45}$ on a subset of hippocampal cells, we recovered the radial-glia-like (RGL) to excitatory-neuron developmental lineage. From RGLs to NIPCs only $5.1 \%$ of tested genes showed DIE $(n=73 ; 95 \% \mathrm{CI}=$ $[4.03,6.34])$. However, threefold more did so from NIPCs to GranuleNB $(n=359,95 \% \mathrm{CI}=[15.81,19.10])$ and then from GranuleNB to excitatory neurons $(n=423,95 \% \quad \mathrm{CI}=$ [14.72,17.54]) (Fig. 5a). Gene ontology (GO) analysis ${ }^{46,47}$ revealed isoform changes in the splicing machinery itself in earlier steps, i.e., from RGL to NIPCs (for Hnrnpa2b1, Snrnp70, Srsf2, Srsf5, Srsf6, Srsf7, Rbm3; Fig. 5b). However, as granule neuroblasts matured to excitatory neurons, DIE was associated with synapse formation and axon elongation (Snap25, Snca, Syp, Dbn1, Cdc42, Nptn, Gap43) among others (Fig. 5c, d).

Importantly, many exon inclusion levels are altered in the transition from dentate gyrus (DG) granuleNB to more mature EN in CA1 and CA3. The synaptic Calcium/Calmodulindependent protein kinase II Beta (Camk2b) has enzymatic and structural roles in neuronal plasticity ${ }^{48}$. Embryonic Camk $2 b$ exploits a 3nt addition to exon 11 and exon-12 exclusion, translating an Alanine instead of Valine ${ }^{49}$. The embryonic form (red exon-11 extension, exon-12 exclusion, Fig. 5e) dominates in more immature granuleNB2 compared to granuleNB1. However, this isoform persists infrequently in mature neuronal types (EN1, EN2, and IN1) indicating cell-type specificity during developmental regulation. Moreover, the additional 3 nucleotides (red in exon 11) co-occur with exon 12, which has not been reported. Also, the first alternative exon (exon 9) increases inclusion as cells differentiate. Furthermore, exon 9 coordination with exon 12 defines cell-subtype differences between EN and IN. All three splicing events occur in the actin binding domain of the CaMKII $\beta$ structure and encode several confirmed phosphorylation sites ${ }^{50}$. Thus, exon coordination among distinct cell types could indicate cell-type-specific morphological changes in the actin cytoskeleton, for instance in spine dynamics ${ }^{51}$ (Fig. 5e).

For the post-synaptic Dlgap4 linked to neuropsychiatric disorders ${ }^{52}$ non-neurons and immature neurons predominantly express one exon. However, during neuronal maturation, exon inclusion switches to both exons (Fig. S10a, b). For Nptn, a gene involved in long-term potentiation, glia and immature neurons predominantly express a single 9nt micro-exon. Mature neurons employ an upstream acceptor, adding four amino acids encoding the cytoplasmic domain, likely relevant for protein-protein interactions $^{53,54}$ (Fig S10b).

Hippocampus enriched developmental gene Fibroblast growth factor 13 (Fgfl3) shows neuronal subtype-specific TSS. Fgf13 exhibited high DIE $(\Delta \Pi>0.5)$ across multiple neuronal cell-type and subtype comparisons. Fgf13 reaches peak expression, specifically in HIPP, at our investigated time point (P7) ${ }^{55}$. Fgf13 is a neuronal developmentally regulated gene and lethal when knocked-out ${ }^{56,57}$. A member of the fibroblast growth factor (FGF) superfamily, Fgf13 is one of four FGF family members (Fgf11-14) labeled fibroblast growth factor homologous factors, which-unlike most FGFs-do not have signal sequences, are not secreted, and function intracellularly ${ }^{58}$. Among its intracellular roles are regulation of voltage-gated sodium channels ${ }^{59-61}$, rRNA transcription $^{62}$, and microtubule stabilization ${ }^{55}$. Of the various $\mathrm{Fg} 13$ isoforms ${ }^{63}$, two isoforms with distinct TSS dominate during brain development ${ }^{55,64}$. We find that $F g f 13$ is particularly alternatively spliced between excitatory and inhibitory neurons (Fig. 5f). The downstream-TSS isoform, Fgf13-S, is the major HIPP isoform across all excitatory types, and immature inhibitory 


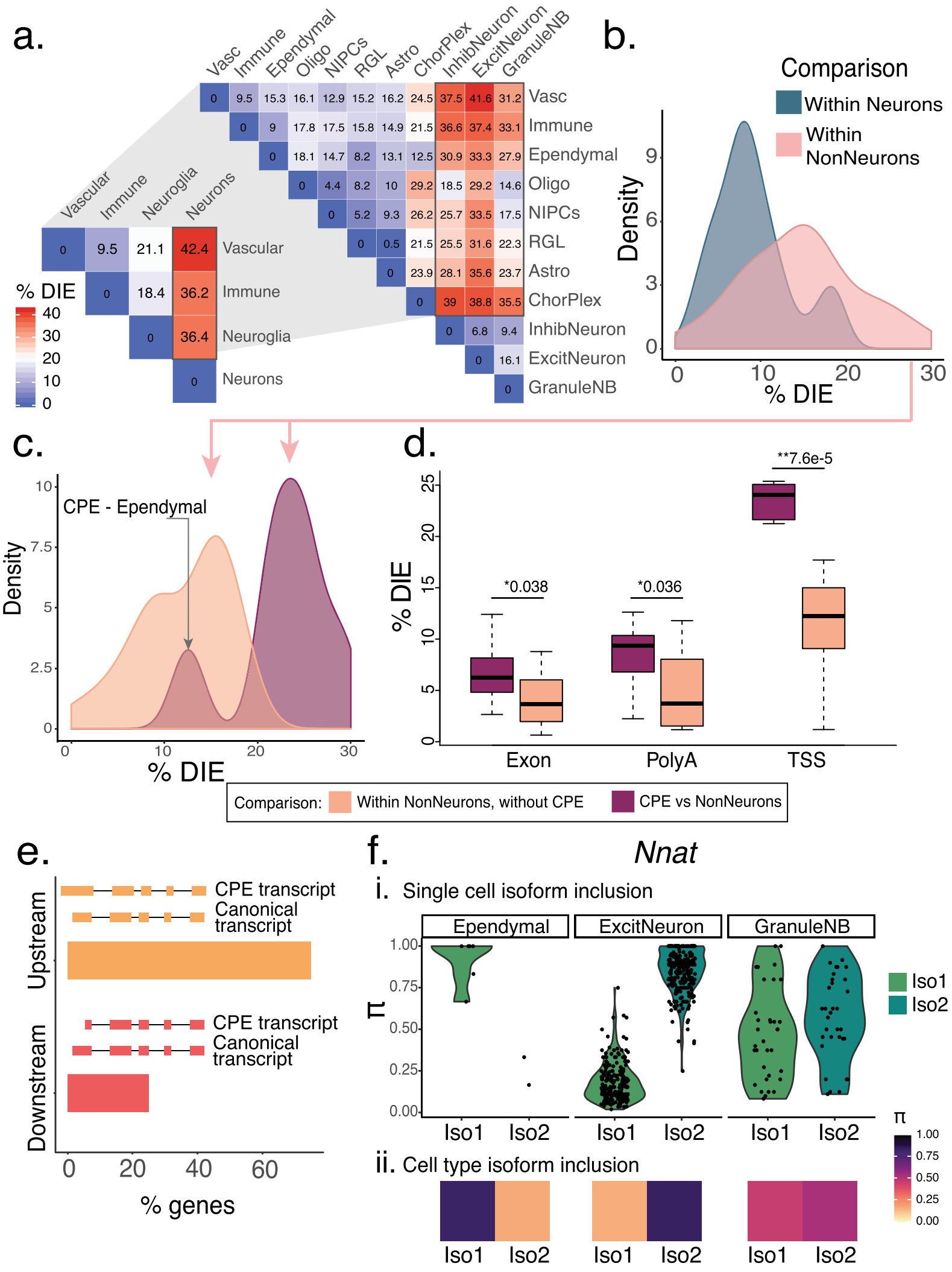

types. Conversely, the upstream-TSS isoform, Fgf13-VY, is partially seen in DG neuroblasts and dominates in inhibitory interneuron classes. This was confirmed using Basescope analysis with probes designed for excitatory and inhibitory neuron marker genes, and separate probes for the S and VY isoforms. We find that the $S$ isoform (pink) co-localizes with excitatory neurons (green, Neurod6) and not inhibitory neurons (green, Gad2), whereas the VY isoform (pink) co-localizes with the inhibitory neurons but not the excitatory neurons (Fig. $5 \mathrm{~g}$, Methods). The isoforms also differ in subcellular localization: reflecting its role in regulating ribosomal biogenesis ${ }^{62}$, Fgf13-S is primarily localized to the nucleolus, whereas Fgf13-VY is present throughout the cytoplasm, consistent with its known role in regulating voltagegated sodium channels ${ }^{60}$ (Fig. $5 \mathrm{~h}$ ). 
Fig. 4 Choroid plexus epithelial cells (CPEs) generate distinct isoforms predominantly through alternative TSS. a Triangular heatmap representing percentage of significant DIE in pairwise comparisons at two levels of granularity. At the broad level: pairwise comparisons of neurons, non-neurons, immune cells, and vascular cells. Zooming in, at the narrow level: neuronal and non-neuronal categories are broken up into their constitutive cell-subtypes. b Density plot of percentage of significant DIE in pairwise comparisons from 4A, broken down by two categories: within neurons and within non-neurons. c Density plot showing DIE within non-neurons (pink region, Fig. 4b) broken up further by comparisons that either include (purple) or exclude (orange) choroid plexus epithelial (CPE) cells. $\mathbf{d}$ Boxplots of percentage significant genes in non-neuronal comparisons including and excluding the CPE, broken down by part of the transcript (TSS/splice-site/PolyA) responsible for splicing changes. $X$-axis indicates the part of the transcript assessed. $P$-values were calculated using a two-sided Wilcoxon rank sum test $\left({ }^{\star \star \star} p<10^{-15},{ }^{\star \star} p<0.001,{ }^{\star} p<0.05\right)$. Data are represented as boxplots where the middle line is the median, the lower and upper hinges correspond to the first and third quartiles, the upper whisker extends from the hinge to the largest value no further than $1.5 \times I Q R$ from the hinge (where IQR is the inter-quartile range) and the lower whisker extends from the hinge to the smallest value at most $1.5 \times I Q R$ of the hinge. Please see function geom_boxplot in R (ggplot2). e Barplot showing the percentage of genes (n-90) for which the CPE transcripts are either upstream or downstream of non-CPE transcripts. $\mathbf{f}$ Percent inclusion (П) of two Nnat isoforms across three cell-types: ependymal cells, excitatory neurons, and granule neuroblasts i. Violin plots of $\Pi$ values of each isoform per single cell ii. $\Pi$ values per isoform across all single cells.

Synaptic genes associated with vesicle transport show splicing differences in developing hippocampal neurons. Many synaptic genes have low expression in developing HIPP. However, sufficiently expressed ( $>50$ reads) genes' isoforms distinguish neuronal subtypes. Two mutually exclusive exons define two isoforms (Snap25-a, Snap25-b) for Snap25, involved in endocytosis. Concordant with the literature ${ }^{65-67}$, our CA3 excitatory neurons (EN3) have a higher proportion of Snap25-b transcripts than in CA1 and subiculum (EN1, EN2). However, our GranuleNBs have higher abundance of Snap25-a whereas mature excitatory neurons switch to Snap25-b. Interestingly, interneuron precursors and Cajal-Retzius cells (IN1,IN3) rely more on Snap25b than their excitatory counterparts, and thereby seem to rely more heavily on larger primed vesicle pools ${ }^{68}$ (Fig. S11b). Alternatively, interneurons could switch from Snap25-a to Snap25-b before excitatory neuron development.

We also observe synaptic genes with key splicing differences between neurons and non-neurons. Clathrin light chain A and B $(C l t a, C l t b)$ work alongside Epsin 1 in vesicle-mediated endocytosis $^{69}$. For all three genes, exon inclusion distinguishes neurons from non-neurons and granuleNB from mature EN. For Clta, an additional exon distinguishes neuronal subtypes (Fig S12a, b). The neuronal specific insertions in the clathrin light chain may influence the association with the slow axonal transport of clathrin $^{70}$.

Slide isoform sequencing (sliso-Seq) to delineate spatial localization of splicing changes. To ground our observations in a spatial sense, we generated 10X Genomics Visium spatial transcriptomics data from a P8 sagittal section. Alignment with single-cell short-read HIPP data confirmed the spatial localization of excitatory neurons in the CA regions and subiculum (Fig. 6a), and alignment with the PFC data confirmed the excitatory precursors in distinct cortical layers (Fig. 6b). We then devised a long-read sequencing approach for spatial transcriptomics (Methods). To validate regional specificity of isoform expression using orthogonal techniques, we correlated $\Delta \Psi$ values between composite HIPP and composite PFC single-cell data with $\Delta \Psi$ values of hippocampus vs. cortex using long-read sequencing. Based on single-cell data, we focused on 40 exons with regionspecific splicing patterns and without alternative acceptors/ donors (Methods). Overall, we found strong concordance: for $85 \%$, both single-cell and spatial HIPP vs. PFC splicing differences point in the same direction (Bernoulli probability $<=3.5 \mathrm{e}$ 06) (Fig. 6c). Additionally, we confirmed neurodevelopmental exon inclusion switches in $\mathrm{Pkm}$ and Clta, where the non-neuronal and developmental exons from the single-cell data were enriched in the DG and in the choroid plexus of the spatial data (Fig. S12, Fig. 6d). For Snap25, the neurodevelopmental switch from Snap25-a to Snap25-b in single-cell data (compare Fig. S11b), occurs in a posterior-to-anterior gradient in spatially mapped exons (Fig. 6e). This supports the idea that the microenvironment can dictate brain-region-specific splicing for some genes. Also, the hypothesis that interneurons selectively switch isoforms before excitatory neurons seems unlikely.

\section{Discussion}

Temporal and anatomic differences in alternative splicing are implicated in developmental changes in molecular function ${ }^{71}$. Building on the short-read investigation of cell-type-specific alternative splicing in the brain ${ }^{72}$, our data enable the illumination of full-length isoform regulation across cell types. We endeavored to generate cell-type, brain-region, and age-specific maps of AS to understand functional consequences of differential isoform expression (DIE). Our analysis hinges on a single-pergene isoform test that considers a gene's entire isoform repertoire and outperforms exon-based tests. Region-specific isoform testing revealed 395 genes with DIE between hippocampus and PFC, partially caused by isoforms novel to GENCODE. Manual validation by the GENCODE team using rigorous metrics lends credibility to this novel isoform detection. Thus, filtering these isoforms using bulk short reads, CAGE and polyA-site data provides a mechanism to automate genome annotation for isoforms.

We defined cell types underlying DIE between brain regions. In most cases and levels of granularity, we identified a finer cell type explanation of DIE, including the altered part of the transcripts. Despite inhibitory interneurons in HIPP and PFC migrating in from a common origin ${ }^{31}$, we observe a signature of coordinated DIE in excitatory and inhibitory neurons for a gene subset. Such gene subsets with coordination across cell types were observed at all investigated levels. Microexons are linked to neuronal cell types for their regulatory function ${ }^{1,41}$, however, in the case of Nsflic we find that their expression is not limited to neurons but instead exhibits regional regulation by being expressed only in HIPP. Thus, brain region can override cell-type specificity for a subset of genes, which may explain region-specific sQTLs ${ }^{21}$. The theoretical possibility that brain-region DIE in bulk arises purely from cell-type abundance or transcriptional activity differences is rarely observed. However, to which extent these observations persist in case-control studies of disease, across adult brain regions, and across species requires further studies.

Our results indicate that understanding the cell-type basis of brain-region-specific DIE requires a thorough understanding of cell-type-specific DIE within each brain region. This further warrants a brain-wide map of isoform expression at a single-cell level. Within the brain, isoform diversity in non-neuronal cell types has attracted less attention than in their neuronal counterparts. However, we find that non-neuronal cell types exhibit high pairwise DIE. This may be partially due to the functional 

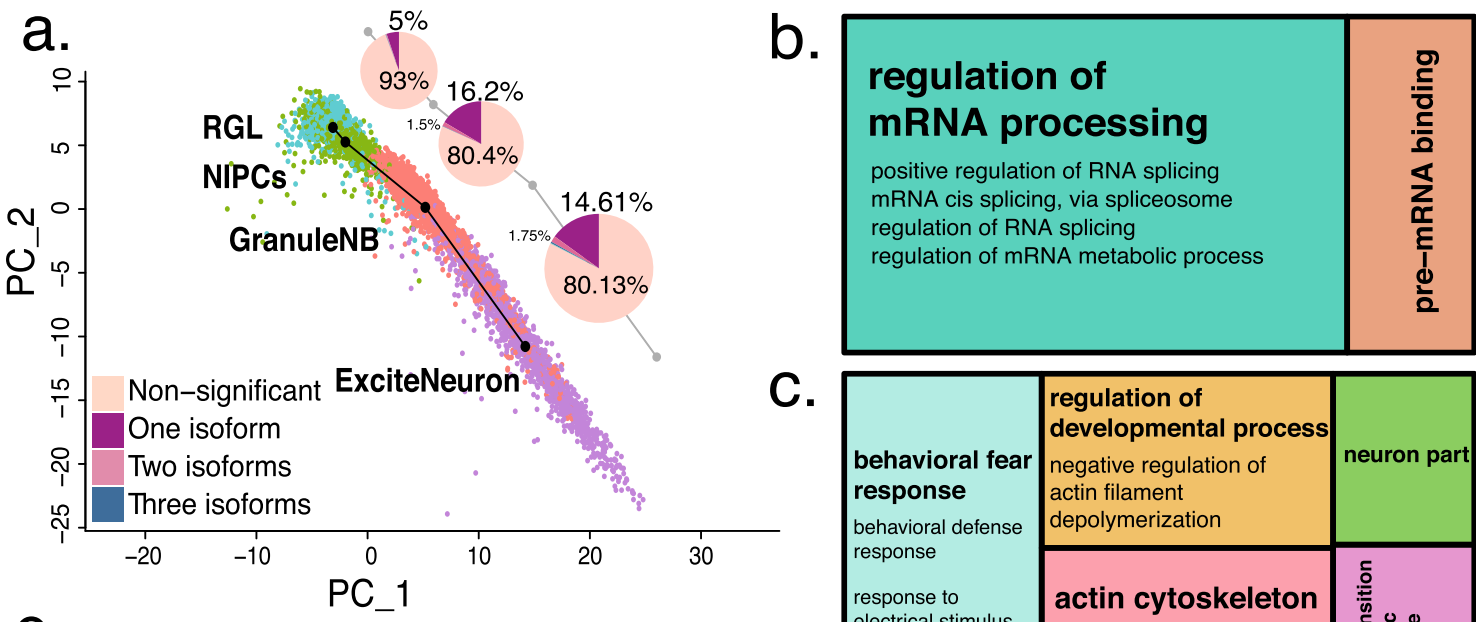

e.
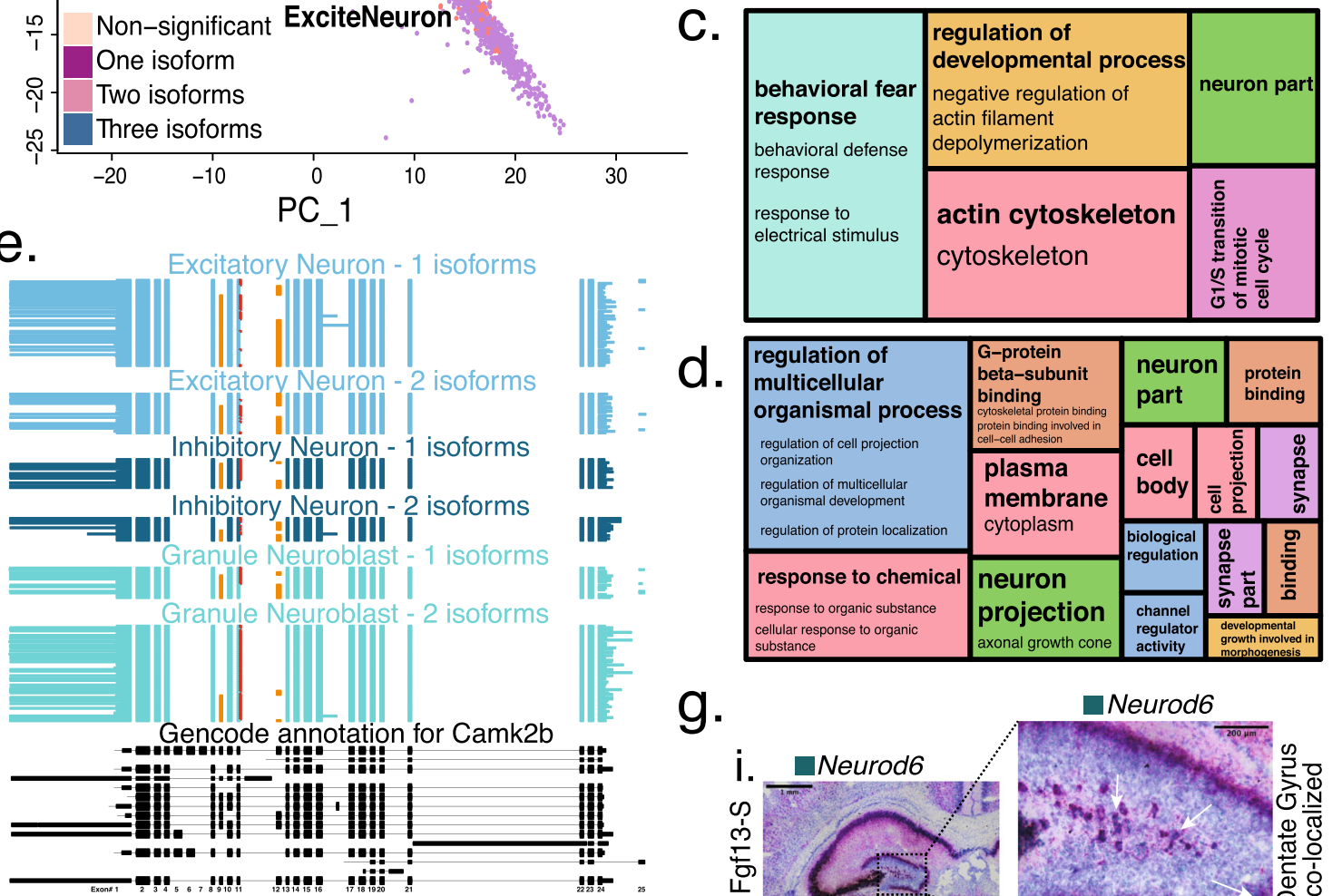

9.

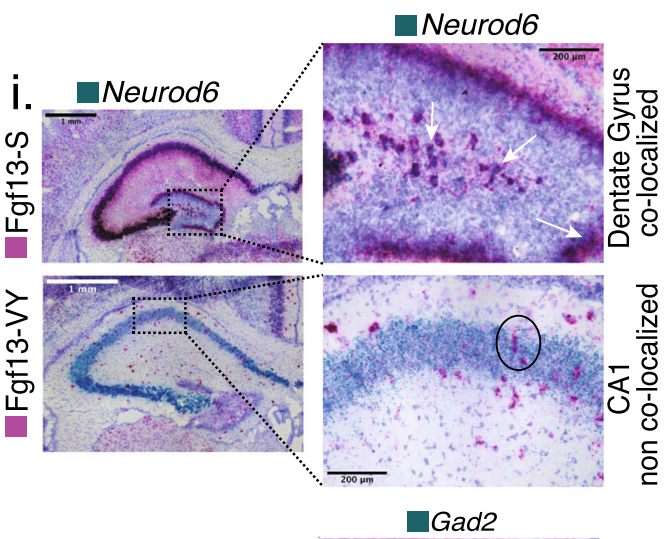

h.

f. $F g f 13$
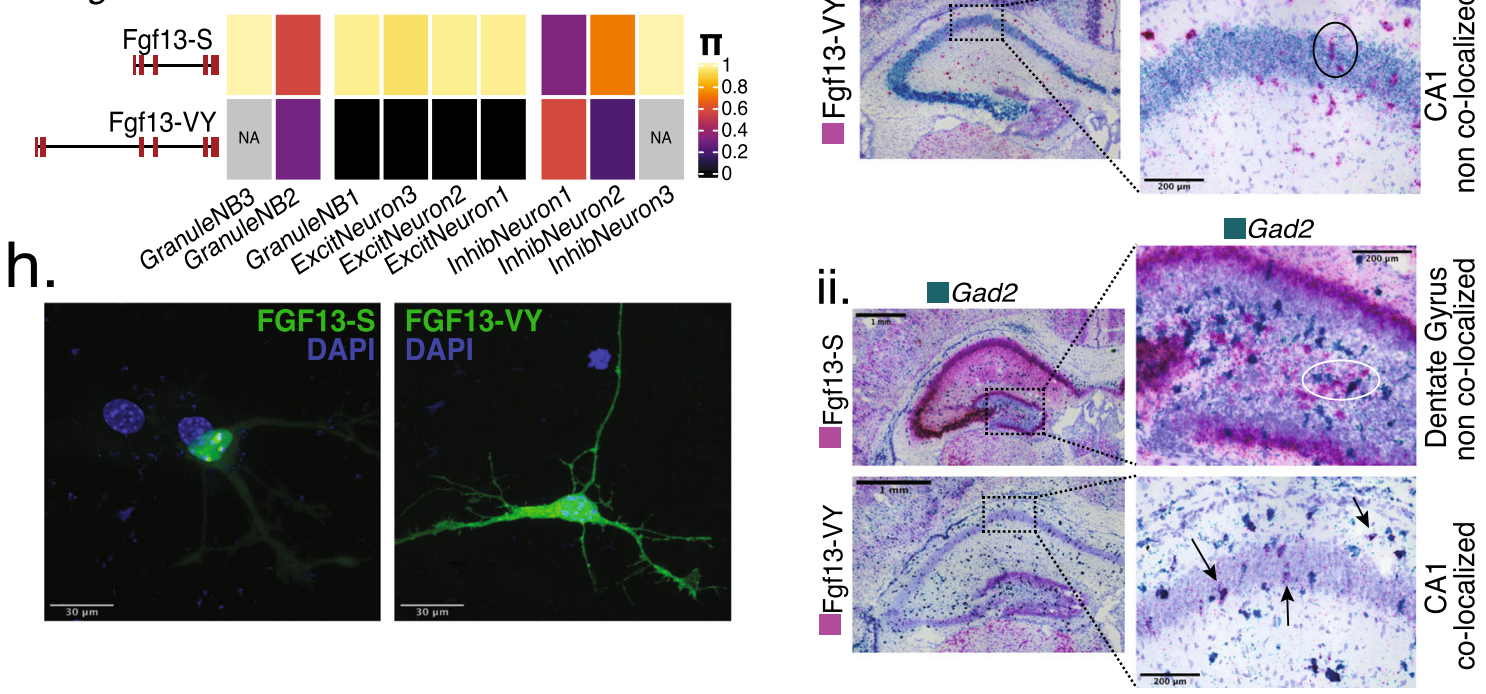

diversity of non-neuronal cells, or due to origins from different stem cell populations. Importantly, excitatory neurons and astrocytes which originate from the same stem cell population but are functionally distinct show high DIE (35.8\%). However, the excitatory neuron population also shows high DIE with Cajal-Retzius cells (18\%) despite both originating from different stem cell populations but functioning as neurons. Among the non-neurons, choroid plexus epithelial cells show particularly large differences from other non-neuronal (but also neuronal) cell types. Surprisingly this observation is largely caused by CPEspecific choices of predominantly upstream TSSs. This raises the question whether other highly specialized cell types in other brain areas exhibit similar complex alternative transcriptome mechanisms. 
Fig. 5 Relative isoform expression differences during development reflect dynamic changes in function. a Splicing changes seen at every transition step of neuronal differentiation trajectory. Pie chart indicates the number of isoforms needed to be considered in order to reach the $|\Delta \Pi| \geq 0.1$ threshold for a gene to be considered significantly DIE. b Treemap of condensed gene ontology (GO) terms for the transition from RGL to NIPCs in the neuronal differentiation trajectory, with size of boxes corresponding to number of significant terms associated with the GO category. c Treemap of condensed GO terms for the transition from NIPCs to GranuleNB (GNB1, GNB2, GNB3). d Treemap of condensed GO terms for the transition from NIPCs to Excitatory Neurons (EN1, EN2, EN3). e Hippocampal cell-type specific isoform expression in Camk2b gene. Each horizontal line in the plot represents a single transcript colored according to the cell-type it is represented in. Orange exons represent alternative inclusion, red extension of exon 11 represents a 3 nt addition. $\mathbf{f} \Pi$ value of the $S$ and $V Y$ isoforms for Fgf13 gene across hippocampal neuronal cell types. $\mathbf{g}$ Basescope (in situ hybridization) images ( $N=1$, repeated for 2 technical replicates). Data showing Fgf13-S and Fgf13-VY isoform expression (pink stain) in the hippocampus, with simultaneous staining for excitatory neurons (Neurod6-green) and inhibitory neurons (Gad2 - green). Scale bar, $1 \mathrm{~mm}$. Each plot features enlargements of the dentate gyrus for the $\mathbf{S}$ isoform, and CA1 region for the VY isoform with arrows indicating co-localization while circles indicate lack of co-localization. Scale bar, $200 \mu \mathrm{m}$. $\mathbf{h}$ Representative images of subcellular localization of overexpressed GFP-tagged Fgf13-S isoform in nucleolus and GFP-tagged Fgf13-VY isoform in cytoplasm. ( $N=3,20-30$ GFP + neurons examined per transfection). Scale bar, $30 \mu \mathrm{m}$.

We notice the sheer functional diversity that AS lends to the splicing machinery, synaptic plasticity, and vesicle-mediated endocytosis. This motivates further investigation linking a spliceosomal-gene isoform to the splicing of its target gene, as well as the isoform state of synaptic genes in neuronal subtypes. The evidence for isoforms adding to cellular diversity is further bolstered by long-read clustering, which yields coherent cell-types albeit with discrepancies, which could be partially due to the sparsity of the isoform matrix due to lower long-read throughput. Alternatively, cell states could be defined by an isoform expression program that does not correspond to $3^{\prime}$ seq-based cell-type definition, highlighting the need for factoring in isoform expression to re-define traditional transcriptional cell-types.

Finally, we have devised the method of slide-isoform sequencing (slISO-Seq), which employs spatial transcriptomics and long-read sequencing. This allows anchoring the above results in a spatial view of the brain and reveals important biology such as the brain-wide coordination of the Snap25 isoform switch. Looking forward, the integration of long-read single-cell and spatial technologies allows for the possibility of constructing 3dimensional maps of isoform expression at single-cell resolution.

In summary, these results present a detailed view of cell-typespecific full-length isoforms across brain regions, bringing us closer to a comprehensive isoform map of the brain. The software generated here is employable in a much larger setting and available as an R-package. It extends to case-control studies, broad sample comparisons, and spatially anchored comparisons beyond mouse or brain.

\section{Methods}

Ethics statement. All experiments were conducted in accordance with relevant NIH guidelines and regulations, related to the Care and Use of Laboratory Animals tissue. Animal procedures were performed according to protocols approved by the Research Animal Resource Center at Weill Cornell College of Medicine.

\begin{abstract}
Animals and tissue isolation. C57BL/6NTac $(n=6)$ female pups were quickly decapitated. For the single-cell experiments (P7; Rep1 $n=1$, Rep2 $n=2$ ), the brains were removed and placed on a stainless-steel brain matrix for mouse (coronal repeatable sections, $1 \mathrm{~mm}$ spacing), and the prefrontal cortex and hippocampus were dissected ${ }^{73}$ in cold PBS solution (Fig. S13a-f). Brain tissues from both hemispheres were pooled in one sample. After dissection, tissues were snap frozen in dry ice until processing. For the $10 \mathrm{X}$ Visium spatial experiment (P8; $n=$ 1) brains were fresh-frozen and embedded in OCT. For the Basescope (P7; $n=2)$ experiments, the brains were transcardially perfused, immersion fixated and cryoprotected (15\% and 30\% Sucrose in phosphate-buffered saline) each over night before being embedded in OCT. Mice were housed in an air-conditioned room at $65-75^{\circ} \mathrm{F}\left(\sim 18-23^{\circ} \mathrm{C}\right)$ with $40-60 \%$ humidity and a 12-h-light, 12-h-dark cycle. All animal experiments were approved by the institutional animal care and use committee (IACUC) of Weill Cornell Medicine
\end{abstract}

Tissue disassociation. Following recommendations from 10x Genomics (Cat\#CG00055 Rev C) dissected hippocampus and prefrontal cortex tissue was placed into $2.5 \mathrm{ml}$ Hibernate E/B27/GlutaMax medium (BrainBits cat\#HEB) at Room Temp until all samples were dissected. HEB medium was removed and replaced with $2 \mathrm{ml}$ of $2 \mathrm{mg} / \mathrm{ml}$ activated papain (BrainBits cat\#PAP) then incubated for $25 \mathrm{~min}$ at $37^{\circ} \mathrm{C}$ with gentle mixing. After allowing tissue to settle, papain was removed and replaced with $2 \mathrm{~mL}$ fresh HEB medium and tissue was gently triturated 15-20 times using a wide-bore pipette tip and tissue left to settle. Supernatant was taken and filtered using a $30 \mu \mathrm{m}$ cell strainer (Miltenyi Biotec cat\#130-041-407) into a collection tube. To the remaining tissue, another $2 \mathrm{ml}$ of fresh HEB medium was added and then triturated with a regular $1 \mathrm{ml}$ pipette tip an additional 10-15 times until tissue was completely disassociated. Supernatant was taken and filtered through a $30 \mathrm{um}$ cell strainer and added to the collection tube. Supernatant was then centrifuged at $400 \mathrm{rcf}$ for $2 \mathrm{~min}$. The cell pellet was re-suspended in $1-3 \mathrm{ml}$ of neuronal culture medium NbActivl (BrainBits cat\#Nbactiv1) depending on cell pellet size, filtered through a $30 \mu \mathrm{m}$ cell strainer (Miltenyi Biotec cat\#130-041-407), and was subsequently diluted to 1500 cells/ $\mu$ in NbActivl for capture on the $10 \mathrm{x}$ Genomics Chromium controller.

10x Genomics single-cell capture. The disassociated cells were captured on the 10x Genomics Chromium controller according to the Chromium Single Cell $3^{\prime}$ Reagent Kits V2 User Guide (10x Genomics CG00052 Rev F) with the following modification. PCR cycles were increased, from the recommended ten cycles for recovery of 8000 cells, to 16 cycles to target a yield of cDNA enabling simultaneous Illumina and PacBio library preparation.

Illumina and Pacific Biosystems library preparation. Illumina library preparation was performed using $100 \mathrm{ng}$ of amplified cDNA following the Chromium Single Cell 3' Reagent Kits V2 User Guide (10x Genomics CG00052 Rev F) reducing final indexing PCR cycles to ten cycles from the recommended 14 cycles to increase library complexity. Sequencing for Replicate 1 was performed on HiSeq4000 according to 10x Genomics run mode, for Replicate 2, sequencing was performed on a NovaSeq S1 flowcell also following 10x Genomics run mode and Bulk RNA-Seq was performed on the Illumina NextSeq 500 with a 150 PE run mode. PacBio library preparation was performed with $500 \mathrm{ng}$ of amplified cDNA using SMRTbell Express Template Prep Kit V2.0 (PacBio cat\#PN: 100-938-900) to obtain Sequel II compatible library complex and was sequenced on a total 24 Sequel I SMRTcells with a run time of $10 \mathrm{~h}$ and 20 Sequel II SMRTcells with a run time of $30 \mathrm{~h}$ across samples and replicates.

Modification of 10x Visium Illumina library preparation. Illumina compatible libraries were made from Visium-derived cDNA using a modified protocol derived from NEB Ultra II DNA FS kit (NEB \#E6177). Visium-derived spatial cDNA was diluted to $100 \mathrm{ng}$ in $26 \mu \mathrm{l}$ and combined with $7 \mu \mathrm{l}$ NEBNext Ultra II FS Reaction Buffer and $2 \mu \mathrm{l}$ NEBNext Ultra II FS Enzyme Mix and incubated at $37^{\circ} \mathrm{C}$ for 15 min, $65^{\circ} \mathrm{C}$ for $30 \mathrm{~min}$ to obtain fragmented, end-repaired, and A-Tailed cDNA. Samples were then subjected to double-sided size selection by following the Beckman Coulter SpriSelect (Cat\# B23318) protocol with an initial ratio of $0.6 \mathrm{x}$ SpriBeads. Supernatant was then taken and additional SpriBeads added for a final ratio of $0.8 \mathrm{x}$ eluting to $35 \mu \mathrm{l} \mathrm{EB}$ Buffer. Adaptor ligation using $10 \mathrm{x}$ Genomics protocol was performed by combining $2.5 \mu \mathrm{l}$ 10x Adaptor Mix with $30 \mu \mathrm{l}$ NEBNext Ultra II Ligation Master Mix and $1 \mu$ l of NEBNext Ligation Enhancer with the previously end-repaired cDNA and incubated at $20^{\circ} \mathrm{C}$ for $15 \mathrm{~min}$. Single-sided SpriBead cleanup was performed using a $0.8 \mathrm{x}$ ratio and eluted in $15 \mu \mathrm{l}$ EB Buffer. Finally cDNA library was amplified by combining adaptor ligated cDNA with $5 \mu \mathrm{l}$ 10x Genomics i7 Barcoded primer and $5 \mu \mathrm{l}$ of 1:5 diluted 10x Genomics SI Primer and $25 \mu \mathrm{l}$ NEBNext Ultra II Q5 Master Mix and cycled with the following thermocyler profile: $98^{\circ} \mathrm{C} 30 \mathrm{~s}, 12$ cycles of $98^{\circ} \mathrm{C} 20 \mathrm{~s}, 54^{\circ} \mathrm{C} 30 \mathrm{~s}, 72^{\circ} \mathrm{C} 30 \mathrm{~s}$, then final extension of $65^{\circ} \mathrm{C}$ for $5 \mathrm{~min}, 10^{\circ} \mathrm{C}$ hold. Amplified library was again subjected to a double-sided size selection using an initial ratio of $0.6 \mathrm{x}$ SpriBeads, supernatant was then taken and additional SpriBeads added for a final ratio of $0.8 \mathrm{x}$ eluting to $35 \mu \mathrm{l}$ EB Buffer. Illumina Libraries were then checked for quality and sequenced on an Illumina NextSeq500 instrument according to guidelines. 


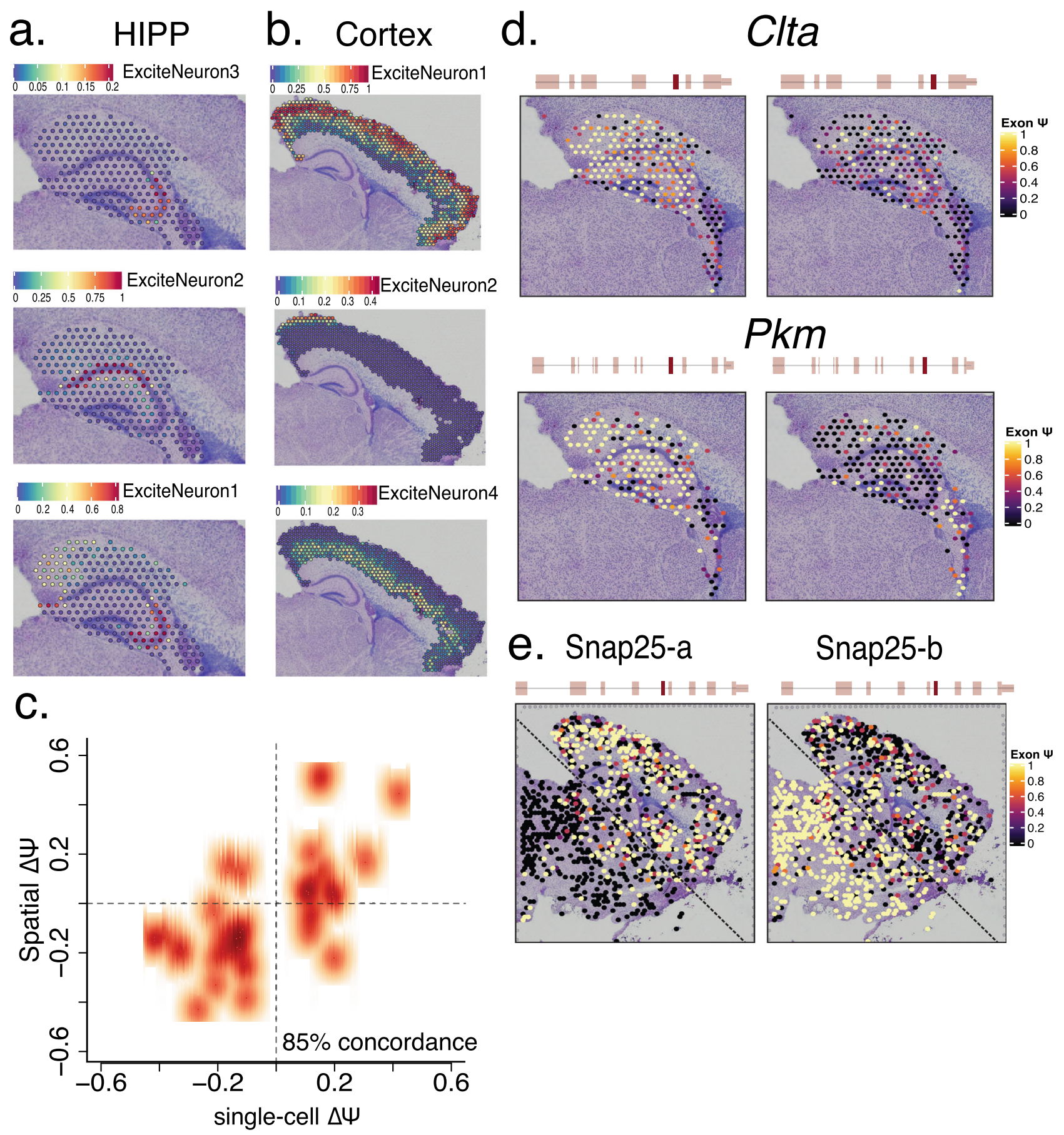

Fig. 6 Slide isoform sequencing (sl-ISO-Seq) confirms spatial localization of splicing changes. a Spatial localization of hippocampal single-cell excitatory neuron subtypes using gene-expression similarities to HIPP short reads. Color of spot indicates percentage of transcripts corresponding to indicated cell type. $\mathbf{b}$ Spatial localization of cortical excitatory neuron subtypes using gene-expression similarities to PFC short reads. Color of spot indicates percentage of transcripts corresponding to indicated cell type. c Scatter plot of the $\Delta \Psi$ between HIPP and PFC from the single-cell data, and the $\Delta \Psi$ between HIPP and cortex from 10X Visium spatial data $\left(r^{2}=0.6\right)$. d Spatial distribution of alternatively spliced exons in Clta and Pkm genes in the hippocampal and choroid plexus regions. Color of spot indicates $\Psi$ values for each exon. e Spatial distribution of two mutually exclusive, alternatively spliced exons in Snap 25 gene across the whole slide. Color of spot indicates $\Psi$ values for each exon.

Size selection of Visium cDNA using blue pippin for Oxford Nanopore. Visium-derived cDNA was size-selected using Sage Sciences Blue Pipin instrument to obtain cDNA fragments $800 \mathrm{bp}$ to $6000 \mathrm{bp}$ for efficient sequencing on Oxford Nanopore PromethIon Instrument. Samples were diluted to $1000 \mathrm{ng}$ in $30 \mu \mathrm{l}$ of TE buffer and combined with $10 \mu \mathrm{l}$ of Sage Loading Solution before loading into one lane of a $0.75 \%$ Agarose Blue Pippin Cassette (Cat\# BLF7510). Samples were then separated according to protocol for a target range of 800-6000 bp, and target elution retrieved after $12 \mathrm{~h}$. Samples were then cleaned by Beckman Coulter
SpriSelect Beads (Cat\# B23318) using a 0.8x ratio and eluted $50 \mu \mathrm{l}$ Nuclease Free Water. Size-distribution was checked using Agilent Fragment Analyzer Large Fragment Kit (Cat\# DNF-464-0500).

PromethION library preparation and sequencing of Visium cDNA. Oxford Nanopore compatible library was produced using $350 \mathrm{ng}$ of either Sage Blue Pippin size-selected cDNA or non-selected cDNA derived from 10x Genomics Visium 
following the Genomic DNA by Ligation protocol (SQK-LSK109) from Oxford Nanopore with the following modifications. End-repair was carried out omitting NEBNext FFPE DNA Repair and incubations extended to $10 \mathrm{~min}$ at $20^{\circ} \mathrm{C}$ and 10 min at $65^{\circ} \mathrm{C}$. Loading inputs on the PromethION was increased to $150 \mathrm{fmol}$ and sequenced for $20 \mathrm{~h}$, and basecalling was done using Guppy (3.2.10).

Total hippocampus and PFC short-read Illumina library preparation. Illumina compatible libraries were produced from 1250 ng total RNA using NEBNext Ultra II RNA Library Prep Kit (NEB Cat\#E7770S) following manufactures protocol with the following modifications. Target insert size was $450 \mathrm{bp}$ for compatibility with paired end $150 \mathrm{bp}$ sequencing mode. Number of PCR cycles was reduced to 6 to limit the effect of PCR aberrations on the final library. Sequencing was performed on the Illumina NextSeq 500 instrument.

Generation of circular consensus reads. Using the default SMRT-Link parameters, we performed circular consensus sequencing (CCS) with IsoSeq3 with the following modified parameters: maximum subread length $14,000 \mathrm{bp}$, minimum subread length $10 \mathrm{bp}$, and minimum number of passes 3 .

\section{Primary hippocampal culture and transfection. Primary dissociated hippo-} campal cultures were prepared as previously described ${ }^{74}$, with minor modifications. Briefly, the hippocampus from P0 mouse pups was dissected on ice, digested with $0.25 \%$ trypsin for $30 \mathrm{~min}$ at $37^{\circ} \mathrm{C}$ with DMEM (Sigma), and dissociated into single cells by gentle trituration. The cells were seeded at a density of $2.5-3.0 \times 10^{5}$ cells per coverslip in Neurobasal-A (Sigma) supplemented with $10 \%$ (vol/vol) heatinactivated FBS onto coverslips previously coated with $50 \mu \mathrm{g} / \mathrm{mL}$ poly-D-lysine (Sigma) overnight at $4{ }^{\circ} \mathrm{C}$ and $25 \mu \mathrm{g} / \mathrm{mL}$ laminin (Sigma) for $2 \mathrm{~h}$ at $37^{\circ} \mathrm{C}$. The cells were maintained in a humidified incubator in $5 \% \mathrm{CO}_{2}$ at $37^{\circ} \mathrm{C}$. After $24 \mathrm{~h}$, the medium was replaced with Neurobasal-A supplemented with 2\% B27 (Invitrogen), $1 \% \mathrm{FBS}, 25 \mu \mathrm{M}$ uridine, and $70 \mu \mathrm{M} 5$-fluorodeoxyuridine. After 6 days of in vitro (DIV) culture, the neurons were transiently transfected with $0.2 \mu \mathrm{g}$ of plasmid DNA per coverslip using calcium phosphate. One day after transfection cultured hippocampal cells were fixed for $30 \mathrm{~min}$ with $4 \%$ paraformaldehyde, washed three times with PBS, and incubated for $5 \mathrm{~min}$ in DAPI solution. Imaging was performed with a Zeiss LSM 880 Laser Scanning Confocal Microscope using an oil immersion $63 \times$ objective. All images were collected at a 2,048 $\times 2,048$-pixel resolution. EGFP fusion constructs were generated as previously described ${ }^{74}$.

Alignment of bulk short-read data for junction validation. Illumina short reads for HIPP and PFC were aligned to the reference genome (mm10) using STAR using the following parameters:

-outFilterMultimapNmax 1 -outFilterIntronMotifs RemoveNoncanonical -outFilterMismatchNmax 5 -alignSJDBoverhangMin 6 -alignSJoverhangMin 6 -outFilterType BySJout -alignIntronMin 25 -alignIntronMax 1000000 -outSAMstrandField intronMotif -outSAMunmapped Within -runThreadN 32 -outStd SAM -alignMatesGapMax 1000000

\section{Alignment of single-cell short read data and analysis. The 10x cellranger} pipeline (version 3.0.0) was run on the raw Illumina sequencing data to obtain single-cell expression matrices. For replicate 1, the raw expression matrices obtained through cellranger were used along with the DropletUtils package $(\mathrm{v}$ $1.6 .1)^{75}$ to acquire 'eligible' barcoded single cells (FDR $<=0.001$ ) with UMI counts that fell below cellranger's filtering cutoff. These barcodes were incorporated into new matrices for importing into Seurat (v 3.1). For both hippocampal replicates and the first PFC replicate, cells that had unique gene counts over 5000 or less than 700 , and greater than $20 \%$ mitochondrial gene expression were removed from further analysis. To adjust for the lower mean reads/cell for the second PFC replicate, the cutoff for minimum number of genes per cell was lowered to 350 . Filtering on these parameters yielded 14,433 single cells for the hippocampus across two replicates, and 10,944 single cells for the PFC. We then used Seurat's "merge" feature $^{76}$ to combine the replicates for each brain region. The number of UMIs, percentage of mitochondrial gene expression were regressed from each cell and then the gene expression matrix was log normalized and scaled to 10,000 reads per cell. Next, we clustered all the cells using 30 principal components (PCs) using the Louvain algorithm with a 0.6 resolution.

Alignment of spatial short read data and analysis. The 10X spaceranger pipeline was run on raw Illumina sequencing data to obtain spatial expression matrices. Seurat's spatial analysis functions were used to obtain gene expression similarity clusters and identify barcodes corresponding to various brain regions.

Integrated analysis with published data to identify cell-types. Published RNASeq P30 mouse brain data from Allen Brain Atlas ${ }^{30}$ was used as a reference to identify cell identities of clusters based on shared gene expression patterns. Since the Allen institute data was generated using the SmartSeq2 protocol, Seurat's integrated anchor feature ${ }^{77}$ was used to align the two datasets and transfer cell-type labels (Fig. S1e, f, S2e, f).
Integrated analysis with spatial transcriptomics data to identify cell-types. P7 HIPP single-cell data was used as a reference to transfer labels onto P8 spatial transcriptomics data in the barcoded region corresponding to the hippocampus using Seurat's integrated anchor feature ${ }^{78}$ using default parameters.

Single-cell trajectory analysis. The velocyto python package $(\mathrm{v} 0.17 .17)^{79}$ was used to obtain.loom files from both replicates of HIPP and PFC single-cell data. After importing the UMAP co-ordinates of the datasets, the $\mathrm{scVelo}^{79}$ package $(\mathrm{v}$ $0.2 .0)$ and tutorial with default parameters were followed to acquire velocity plots (Fig. S1c, d, S2c, d). The cells involved in neurogenesis and neuronal differentiation in the dentate gyrus and hippocampus were subsetted based on cell-type identity, and slingshot trajectory analysis ${ }^{45}$ was conducted on its first two principal components in an unsupervised manner (Fig. 5a).

Alignment of Pacific Biosciences long read data. Long read CCS fastqs sequences with PacBio were mapped and aligned to the reference genome (mm10) using STARlong and the following parameters:

-readFilesCommand zcat -runMode alignReads -outSAMattributes NH HI NM MD -readNameSeparator space --outFilterMultimapScoreRange 1 -outFilterMismatchNmax 2000 -scoreGapNoncan -20 -scoreGapGCAG -4 -scoreGapATAC -8 -scoreDelOpen -1 -scoreDelBase -1 -scoreInsOpen -1 -scoreInsBase -1 -alignEndsType Local -seedSearchStartLmax 50 -seedPerReadNmax 100000 -seedPerWindowNmax 1000

-alignTranscriptsPerReadNmax 100000 -alignTranscriptsPerWindowNmax 10000

Alignment of spatial transcriptomics data sequenced using Oxford Nanopore Long reads sequenced on the ONT PromethION were mapped and aligned using minimap2 using the following parameters: -t 20 -ax splice -secondary $=$ no

Filtering of long reads for full-length, spliced, barcoded reads. This was first described in our previous publication ${ }^{80}$ but we outline the process here as well. We first filtered long reads by retaining only alignments with the following procedure. If the read had exactly one alignment and the mapping quality of that alignment was at least 20 (in the sam format mapping quality definition), this alignment was kept. If the read has multiple alignments, but one alignment's mapping quality outscored the maximum of all other mapping qualities for that read by at least 20 the highest scoring alignment was retained. Afterwards, we removed alignments that overlapped annotated ribosomal RNA genes. For spliced alignments, we determined the splice site consensus for each intron in the alignment and only retained, alignments, for which every single intron respected the GT-AG, GC-AG, or ATAC consensus. These alignments we have previously referred to as a consensus-split-mapped molecule (CSMM). For each CSMM, we counted the number of splice sites it shared with each annotated gene. The vast majority of CSMMs share splice sites with exactly one gene. Each CSMM was assigned to the gene with which it shared most splice sites (or all such genes, in the case of a tie). Additionally, we filtered for complete reads using published CAGE peaks and polyA site data ${ }^{80,81}$ by only keeping CSMMs that had start and end sites falling within $50 \mathrm{bp}$ of an annotated CAGE or polyA peak.

Exon count assignment per cell-type. High confidence mapped and aligned reads were processed sequentially and compared to the Gencode M21 gene annotation using an in-house script. A Gencode-annotated exon (Fig. S14a) was considered as being included in the read if both splice junctions were detected by the alignment. Due to the high error rate of ONT sequencing data, a variation of 3 bp was allowed for each splice junction, whereas for PacBio variation allowed was $2 \mathrm{bp}$, and overlapping exons were flagged (Fig. S14b). In cases where a read spanned an exon, but its splice junctions were not detected, the exon was considered excluded (Fig. S14c). Although ONT reads are known to often represent truncated versions of transcripts, terminal exons were counted but were only considered included when covered completely. Partially covered terminal exons were considered neither included nor excluded, and were discarded from the analysis (Fig. S14d). Further, exon counts were aggregated to produce exclusion and inclusion rates for each particular cell group.

Differential isoform tests. For isoform tests, a read was represented by a string denoting the TSS, introns, and polyA-site and each isoform was assigned an ID, with lower numbers corresponding to higher abundances. Next, counts for each isoform ID were assigned to individual cell-types. For each differential abundance test between two categories, genes were filtered out as 'untestable' if reads did not reach sufficient depth ( 25 reads/gene category). For genes with sufficient depth, a maximum of an $11 \times 2$ matrix of counts denoting isoform $\times$ category was constructed with the first ten rows corresponding up to the first ten isoforms, and the last row comprised of collapsed counts from all the other isoforms (if any). Pvalues from a $\chi^{2}$ test were reported per gene, along with a $\Delta \Pi$ value per gene. The $\Delta \Pi$ was constructed as the sum of change in percent isoform ( $\Pi$ ) of the top two isoforms in either positive or negative direction. After these numbers were reported for all testable genes for a comparison, the Benjamini Hochberg $(\mathrm{BH})^{82}$ correction for multiple testing with a false discovery rate of $5 \%$ was applied to return a 
corrected $p$-value. If this FDR $p$-value was $<=0.05$ and the $\Delta \Pi$ was more than 0.1 in one direction, i.e., the change in percent inclusion of one or two isoforms was more than $10 \%$ from one category to the other, then the gene was considered to be significantly differentially spliced.

Differential TSS tests. For each read, we determined the TSS as described above. The counts of each TSS in two conditions (e.g., excitatory and inhibitory neurons) were then summarized in a $n \times 2$ table of counts. As with the isoform tests, this table was reduced to a $11 \times 2$ table where the first ten rows represented the most abundant TSS and the $11^{\text {th }}$ represented all the other TSS. Testing was performed as described above for isoforms.

Differential polyA-site test. For each read, we determined the polyA-site as described above. The procedure for testing was the same as described above for differential TSS.

Differential exon tests. Testing for differential exon inclusion followed a separate framework. For testing differential inclusion between two categories, a $2 \times 2$ table of inclusion and exclusion counts per exon was constructed if it was not constitutively spliced $(0.1<=\Psi<=0.9)$ without considering counts in individual categories. An exon was considered for testing if the expected counts followed the criteria ${ }^{83,84}$ $\frac{\min (\text { rowSums }) \times \min (\text { colsums })}{\text { To }} \geq 5$, and the $p$-value from a $\chi^{2}$ test was reported, along with a $\Delta \Psi$ value. The $\Delta \Psi$ was constructed as the difference in percent spliced in (PSI $/ \Psi$ ) across the two categories. After these numbers were reported for all testable exons for a comparison, since these tests are dependent, the Benjamini Yekutieli (BY) ${ }^{85}$ correction for multiple testing with a false discovery rate of $5 \%$ was applied to return a corrected $p$-value. If this $p$-value was $<=0.05$ and the $\Delta \Psi$ was more than 0.1 i.e., the change in $\Psi$ was more than $10 \%$ between the two categories, then the exon is considered to be significantly differentially included.

\section{Isolating non-overlapping exons from single-cell data for correlation with} spatial ONT data. To confirm region-specific DIE in a technologically orthogonal fashion, we used spatial data sequenced with Oxford Nanopore as opposed to single-cell data sequenced with PacBio. Because of the relatively high error rate, truncated transcripts ( 200 bp difference in average length of ONT vs PacBio), and mis-mapping of overlapping splice sites, we chose to work with exons instead of full-length transcripts. We isolated exons from the single-cell PacBio data that showed region-specific DIE without $p$-value correction and extracted corresponding exons from the spatial data if they satisfied the following condition. If an exon was reported to have alternative donor and acceptor sites, it was only accepted if more than $90 \%$ of reads overlapping the exon mapped to that particular exon. We then used this list of exons to calculate region-specific DIE using spatial exon expression and correlated the $\Delta \Psi$ values with the $\Delta \Psi$ values from the single-cell data. Despite high technical variation, we got high concordance (85\% change in the same direction, $n=40$ ).

Building an enhanced annotation. We isolated polyadenylated, barcoded, and spliced long-reads,

- whose TSS were within 50 bps of a published CAGE-peak ${ }^{80}$

- whose mapped read-end fell within 50 bps of a published polyA-site ${ }^{8}$

- whose intron-chains were inconsistent with any annotated transcript or any truncated version of an annotated transcript ${ }^{86,87}$

- whose internal exons (meaning both splice sites) were each supported by two or more single short reads (with $\geq 2$ splicing events) or paired-end Illumina read pairs from a bulk-sequencing experiment

- whose introns were each supported by two or more spliced Illumina reads from a bulk sequencing experiment

- who could not be interpreted as a truncated version of another such novel isoform

This resulted in an enhanced annotation, in which all added isoforms had a unique, previously absent intron-chain.

These isoforms as well as already annotated isoforms received read counts in each cell types according to the single-cell long-read dataset in each brain region. Novel isoforms intron-chains are by construction (see above) unique; however, annotated isoforms can differ only in their TSS or polyA-site. A long-read was therefore assigned to a GENCODE transcript that minimized the sum of abs (readEndMapping - annotatedIsoformEnd) and abs(readStartMappingannotatedIsoformStart). In the case of a tie, only the divergence at the TSS was considered.

This annotation thus includes counts for thousands of isoforms in different cell types, such as "P7Hipp_OPCs" (representing oligodendrocyte precursor cells from a hippocampus at postnatal day 7) and "P7PFC_OPCs" (representing oligodendrocyte precursor cells from a pre-frontal cortex at postnatal day 7).

Manual validation from the GENCODE team. Novel isoforms were manually verified and integrated into the GENCODE geneset ${ }^{88}$ in accordance with guidelines developed by the HAVANA group for the GENCODE / ENCODE projects ${ }^{89}$.
Novel introns not previously identified within the GENCODE geneset were independently confirmed by intron predictions taken from the Ensembl RNAsed pipeline using ENCODE data ${ }^{90,91}$, whilst novel transcription start sites were validated based on the Cap Analysis of Gene Expression (CAGE) libraries generated by FANTOM ${ }^{92}$. Finally, each new transcript model was assigned a 'biotype' indicative of its presumed functional categorization, with the biotypes matching to those used in GENCODE as described by Frankish et al. ${ }^{88}$.

Validation of cell-type-specific splicing using Basecope Duplex Detection Assay. Target transcriptomic regions for individual isoforms were isolated from the DIE analysis and probes were designed by Advanced Cell Diagnostics (ACD) Postnatal day 7 mice were transcardially perfused, and $12 \mu \mathrm{m}$ brain sections were processed according to the manufacturers recommendation for BaseScope Duplex Detection Assay (Protocol number 323800-USM, Advanced Cell Diagnostics, ACD) with a pre-treatment according to the protocol for fixed, frozen tissue (Protocol number 320534, Advanced Cell Diagnostics, ACD) with only $15 \mathrm{~min}$ of Protease Plus incubation. Images were taken using a Leica DM5500 B with a Leica DFC295 camera.

Reporting summary. Further information on research design is available in the Nature Research Reporting Summary linked to this article.

\section{Data availability}

All data used for this study is publicly available on GEO under the accession token GSE158450. All data supporting the findings of this study are provided within the paper and its supplementary information. All additional information will be made available upon reasonable request to the authors.

\section{Code availability}

The source code for the methods and visualizations are available as functions in an Rpackage $^{93}$ (https://github.com/noush-joglekar/scisorseqr) and processed single-cell and spatial data is available for download at www.isoformAtlas.com

Received: 9 September 2020; Accepted: 27 November 2020; Published online: 19 January 2021

\section{References}

1. Raj, B. \& Blencowe, B. J. Alternative splicing in the mammalian nervous system: recent insights into mechanisms and functional roles. Neuron 87, 14-27 (2015).

2. Wang, E. T. et al. Alternative isoform regulation in human tissue transcriptomes. Nature 456, 470-476 (2008).

3. Nilsen, T. W. \& Graveley, B. R. Expansion of the eukaryotic proteome by alternative splicing. Nature 463, 457-463 (2010)

4. Song, Y. et al. Single-cell alternative splicing analysis with expedition reveals splicing dynamics during neuron differentiation. Mol. Cell 67, 148-161.e5 (2017).

5. Forrest, A. R. R. et al. A promoter-level mammalian expression atlas. Nature 507, 462-470 (2014).

6. Mayr, C. Evolution and biological roles of alternative 3'UTRs. Trends Cell Biol. 26, 227-237 (2016)

7. Sandberg, R., Neilson, J. R., Sarma, A., Sharp, P. A. \& Burge, C. B. Proliferating cells express mRNAs with shortened $3^{\prime}$ untranslated regions and fewer microRNA target sites. Science 320, 1643-1647 (2008).

8. Weyn-Vanhentenryck, S. M. et al. Precise temporal regulation of alternative splicing during neural development. Nat. Commun. 9, 2189 (2018).

9. Nutter, C. A. et al. Cell-type-specific dysregulation of RNA alternative splicing in short tandem repeat mouse knockin models of myotonic dystrophy. Genes Dev. https://doi.org/10.1101/gad.328963.119 (2019).

10. Fagnani, M. et al. Functional coordination of alternative splicing in the mammalian central nervous system. Genome Biol. 8, R108 (2007).

11. Tilgner, H. et al. Comprehensive transcriptome analysis using synthetic longread sequencing reveals molecular co-association of distant splicing events. Nat. Biotechnol. 33, 736-742 (2015).

12. Tilgner, $H$. et al. Microfluidic isoform sequencing shows widespread splicing coordination in the human transcriptome. Genome Res. $\mathbf{2 8}$ 231-242 (2018)

13. Anvar, S. Y. et al. Full-length mRNA sequencing uncovers a widespread coupling between transcription initiation and mRNA processing. Genome Biol. 19, 46 (2018).

14. Bolisetty, M. T., Rajadinakaran, G. \& Graveley, B. R. Determining exon connectivity in complex mRNAs by nanopore sequencing. Genome Biol. 16 204 (2015). 
15. Roy, C. K., Olson, S., Graveley, B. R., Zamore, P. D. \& Moore, M. J. Assessing long-distance RNA sequence connectivity via RNA-templated DNA-DNA ligation. Elife 4, e03700 (2015).

16. Treutlein, B., Gokce, O., Quake, S. R. \& Südhof, T. C. Cartography of neurexin alternative splicing mapped by single-molecule long-read mRNA sequencing. Proc. Natl Acad. Sci. USA 111, E1291-E1299 (2014).

17. Schreiner, D. et al. Targeted combinatorial alternative splicing generates brain region-specific repertoires of neurexins. Neuron 84, 386-398 (2014).

18. Yeo, G., Holste, D., Kreiman, G. \& Burge, C. B. Variation in alternative splicing across human tissues. Genome Biol. 5, R74 (2004).

19. Yang, Y. Y. L., Yin, G. L. \& Darnell, R. B. The neuronal RNA-binding protein Nova-2 is implicated as the autoantigen targeted in POMA patients with dementia. Proc. Natl Acad. Sci. 95, 13254-13259 (1998).

20. McKee, A. E. et al. A genome-wide in situ hybridization map of RNA-binding proteins reveals anatomically restricted expression in the developing mouse brain. BMC Dev. Biol. 5, 14 (2005).

21. Zhang, Y. et al. Regional variation of splicing QTLs in human brain. Am. J. Hum. Genet. https://doi.org/10.1016/j.ajhg.2020.06.002 (2020).

22. Darnell, R. B. RNA protein interaction in neurons. Annu. Rev. Neurosci. 36, 243-270 (2013).

23. Vuong, C. K., Black, D. L. \& Zheng, S. The neurogenetics of alternative splicing. Nat. Rev. Neurosci. 17, 265-281 (2016).

24. Norris, A. D. \& Calarco, J. A. Emerging roles of alternative pre-mRNA splicing regulation in neuronal development and function. Front. Neurosci. 6, $122(2012)$

25. Vaquero-Garcia, J. et al. A new view of transcriptome complexity and regulation through the lens of local splicing variations. Elife 5, e11752 (2016).

26. Gupta, I. et al. Single-cell isoform RNA sequencing characterizes isoforms in thousands of cerebellar cells. Nat. Biotechnol. 36, 1197-1202 (2018).

27. Stuart, T. et al. Comprehensive integration of single-cell data.Cell 177, 1888-1902.e21 (2019).

28. Hochgerner, H., Zeisel, A., Lönnerberg, P. \& Linnarsson, S. Conserved properties of dentate gyrus neurogenesis across postnatal development revealed by single-cell RNA sequencing. Nat. Neurosci. 21, 290-299 (2018).

29. Zeisel, A. et al. Molecular architecture of the mouse nervous system resource molecular architecture of the mouse nervous system. Cell 174, 999-1014 (2018).

30. Yao, Z. et al. A taxonomy of transcriptomic cell types across the isocortex and hippocampal formation. Preprint at https://doi.org/10.1101/2020.03.30.015214 (2020).

31. Miyoshi, G. et al. Genetic fate mapping reveals that the caudal ganglionic eminence produces a large and diverse population of superficial cortical interneurons. J. Neurosci. 30, 1582-1594 (2010).

32. Mayer, C. et al. Developmental diversification of cortical inhibitory interneurons. Nature https://doi.org/10.1038/nature25999 (2018).

33. Krienen, F. M. et al. Innovations present in the primate interneuron repertoire. Nature 586, 262-269 (2020).

34. Zeisel, A. et al. Molecular architecture of the mouse nervous system. Cell 174, 999-1014.e22 (2018)

35. Tasic, B. et al. Adult mouse cortical cell taxonomy revealed by single cell transcriptomics. Nat. Neurosci. 19, 335-346 (2016).

36. Benjamini, Y. \& Yekutieli, D. The control of the false discovery rate in multiple testing under dependency. Ann. Stat. 29, 1165-1188 (2001).

37. Wang, K. Z. Q. et al. PINK1 interacts with VCP/p97 and activates PKA to promote NSFL1C/p47 phosphorylation and dendritic arborization in neurons. eNeuro 5, ENEURO.0466-18 (2018).

38. Shih, Y. T. \& Hsueh, Y. P. VCP and ATL1 regulate endoplasmic reticulum and protein synthesis for dendritic spine formation. Nat. Commun. 7, 11020 (2016).

39. Spilker, C. et al. A Jacob/Nsmf gene knockout results in hippocampal dysplasia and impaired BDNF signaling in dendritogenesis. PLoS Genet. 12, e1005907 (2016).

40. Behnisch, T. et al. Nuclear translocation of Jacob in hippocampal neurons after stimuli inducing long-term potentiation but not long-term depression. PLoS One 6, e17276 (2011).

41. Irimia, M. et al. A highly conserved program of neuronal microexons is misregulated in autistic brains. Cell 159, 1511-1523 (2014).

42. Yamanaka, S. et al. Targeted disruption of the Hexa gene results in mice with biochemical and pathologic features of Tay-Sachs disease. Proc. Natl Acad. Sci. U.S.A. 91, 9975-9979 (1994).

43. Lun, M. P., Monuki, E. S. \& Lehtinen, M. K. Development and functions of the choroid plexus-cerebrospinal fluid system. Nat. Rev. Neurosci. https://doi.org/ 10.1038/nrn3921 (2015).

44. Leppek, K., Das, R. \& Barna, M. Functional 5' UTR mRNA structures in eukaryotic translation regulation and how to find them. Nat. Rev. Mol. Cell Biol. 19, 158-174 (2018).

45. Street, K. et al. Slingshot: cell lineage and pseudotime inference for single-cell transcriptomics. BMC Genomics 19, 477 (2018).
46. Eden, E., Navon, R., Steinfeld, I., Lipson, D. \& Yakhini, Z. GOrilla: A tool for discovery and visualization of enriched GO terms in ranked gene lists. $B M C$ Bioinforma. 10, 48 (2009).

47. Supek, F., Bošnjak, M., Škunca, N. \& Šmuc, T. REVIGO summarizes and visualizes long lists of gene ontology terms. PLoS One 6, e21800 (2011).

48. Borgesius, N. Z. et al. $\beta$ CaMKII plays a nonenzymatic role in hippocampal synaptic plasticity and learning by targeting $\alpha$;CaMKII to synapses. $J$. Neurosci. 31, 10141-10148 (2011).

49. Brocke, L., Srinivasan, M. \& Schulman, H. Developmental and Regional Expression of Multifunctional Ca*+/Calmodulin-Dependent Protein Kinase lsoforms in Rat Brain. J. Neurosci. 75, 6797-6808 (1995).

50. Baucum, A. J., Shonesy, B. C., Rose, K. L. \& Colbran, R. J. Quantitative proteomics analysis of CaMKII phosphorylation and the CaMKII interactome in the mouse forebrain. ACS Chem. Neurosci. 6, 615-631 (2015).

51. Khan, S., Downing, K. H. \& Molloy, J. E. Architectural dynamics of CaMKIIactin networks. Biophys. J. 116, 104-119 (2019).

52. Schob, C. et al. Cognitive impairment and autistic-like behaviour in SAPAP4deficient mice. Transl. Psychiatry 9, 7 (2019).

53. Dagley, L. F. et al. Quantitative proteomic profiling reveals novel regionspecific markers in the adult mouse brain. Proteomics 14, 241-261 (2014).

54. Beesley, P. W., Herrera-Molina, R., Smalla, K.-H. \& Seidenbecher, C. The Neuroplastin adhesion molecules: key regulators of neuronal plasticity and synaptic function. J. Neurochem. 131, 268-283 (2014).

55. Wu, Q. F. et al. Fibroblast growth factor 13 is a microtubule-stabilizing protein regulating neuronal polarization and migration. Cell 149, 1549-1564 (2012).

56. Puranam, R. S. et al. Disruption of Fgf13 causes synaptic excitatory-inhibitory imbalance and genetic epilepsy and febrile seizures plus. J. Neurosci. 35, 8866-8881 (2015)

57. Sinden, D. S. et al. Knockout of the X-linked Fgf13 in the hypothalamic paraventricular nucleus impairs sympathetic output to brown fat and causes obesity. FASEB J. 33, 11579-11594 (2019)

58. Pablo, J. L. \& Pitt, G. S. Fibroblast growth factor homologous factors: new roles in neuronal health and disease. Neuroscientist 22, 19-25 (2016).

59. Wang, C. et al. Fibroblast growth factor homologous factor 13 regulates $\mathrm{Na}+$ channels and conduction velocity in murine hearts. Circ. Res. 109, 775-782 (2011).

60. Pablo, J. L., Wang, C., Presby, M. M. \& Pitt, G. S. Polarized localization of voltage-gated $\mathrm{Na}^{+}$channels is regulated by concerted FGF13 and FGF14 action. Proc. Natl Acad. Sci. U.S.A 113, E2665-E2674 (2016).

61. Yang, L. et al. FGF13 selectively regulates heat nociception by interacting with Nav1.7. Neuron 93, 806-821.e9 (2017).

62. Bublik, D. R. et al. Regulatory module involving FGF13, miR-504, and p53 regulates ribosomal biogenesis and supports cancer cell survival. Proc. Natl Acad. Sci. U.S.A. 114, E496-E505 (2017).

63. Yang, J. et al. FGF13 modulates the gating properties of the cardiac sodium channel Nav1.5 in an isoform-specific manner. Channels 10, 410-420 (2016).

64. Munoz-Sanjuan, I., Smallwood, P. M. \& Nathans, J. Isoform diversity among fibroblast growth factor homologous factors is generated by alternative promoter usage and differential splicing. J. Biol. Chem. 275, 2589-2597 (2000).

65. Bark, C. I., Hahn, K. M., Ryabinin, A. E. \& Wilson, M. C. Differential expression of SNAP-25 protein isoforms during divergent vesicle fusion events of neural development. Proc. Natl Acad. Sci. U.S.A. 92, 1510-1514 (1995).

66. Bark, C. et al. Developmentally regulated switch in alternatively spliced SNAP25 isoforms alters facilitation of synaptic transmission. J. Neurosci. 24 8796-8805 (2004)

67. Bark, I. C. \& Wilson, M. C. Human cDNA clones encoding two different isoforms of the nerve terminal protein SNAP-25. Gene 139, 291-292 (1994).

68. Sørensen, J. B. et al. Differential control of the releasable vesicle pools by SNAP-25 splice variants and SNAP-23. Cell 114, 75-86 (2003).

69. Blue, R. E., Curry, E. G., Engels, N. M., Lee, E. Y. \& Giudice, J. How alternative splicing affects membrane-trafficking dynamics. J. Cell Sci. 131, jcs216465 (2018).

70. Brodsky, F. M. et al. Clathrin light chains: arrays of protein motifs that regulate coated-vesicle dynamics. Trends Biochem. Sci. 16, 208-213 (1991).

71. Nakano, Y., Wiechert, S. \& Bánfi, B. Overlapping activities of two neuronal splicing factors switch the GABA effect from excitatory to inhibitory by regulating REST. Cell Rep. 27, 860-871.e8 (2019).

72. Furlanis, E., Traunmüller, L., Fucile, G. \& Scheiffele, P. Landscape of ribosome-engaged transcript isoforms reveals extensive neuronal-cell-classspecific alternative splicing programs. Nat. Neurosci. 22, 1709-1717 (2019).

73. The Mouse Brain in Stereotaxic Coordinates, Compact - 3rd Edition. https:// www.elsevier.com/books/the-mouse-brain-in-stereotaxic-coordinatescompact/franklin/978-0-12-374244-5 (2020).

74. Pablo, J. L., Wang, C., Presby, M. M. \& Pitt, G. S. Polarized localization of voltage-gated $\mathrm{Na}^{+}$channels is regulated by concerted FGF13 and FGF14 action. Proc. Natl Acad. Sci. U.S.A 113, E2665-E2674 (2016). 
75. Lun, A. T. L. et al. EmptyDrops: distinguishing cells from empty droplets in droplet-based single-cell RNA sequencing data. Genome Biol. 20, 63 (2019).

76. Butler, A., Hoffman, P., Smibert, P., Papalexi, E. \& Satija, R. Integrating singlecell transcriptomic data across different conditions, technologies, and species. Nat. Biotechnol. 36, 411-420 (2018).

77. Stuart, T. et al. Comprehensive integration of single-cell data. Cell 177, 1888-1902.e21 (2019).

78. La Manno, G. et al. RNA velocity of single cells. Nature 560, 494-498 (2018).

79. Bergen, V., Lange, M., Peidli, S., Wolf, F. A. \& Theis, F. J. Generalizing RNA velocity to transient cell states through dynamical modeling. Nat. Biotechnol. 38, 1408-1414 (2020).

80. Lizio, M. et al. Gateways to the FANTOM5 promoter level mammalian expression atlas. Genome Biol. 16, 22 (2015).

81. Herrmann, C. J. et al. PolyASite 2.0: a consolidated atlas of polyadenylation sites from $3^{\prime}$ end sequencing. Nucleic Acids Res. 48, D174-D179 (2019).

82. Benjamini, Y. \& Hochberg, Y. Controlling the false discovery rate: a practical and powerful approach to multiple. Test. J. R. Stat. Soc. Ser. B 57, 289-300 (1995).

83. Cochran, W. G. The $\chi^{2}$ test of goodness of fit. Ann. Math. Stat. 23, 315-345 (1952).

84. Cochran, W. G. Some methods for strengthening the common $\chi^{2}$ tests. Biometrics 10, 417 (1954).

85. Benjamini, Y. \& Yekutieli, D. The control of the false discovery rate in multiple testing under dependency. Ann. Stat. 29, 1165-1188 (2001).

86. Sharon, D., Tilgner, H., Grubert, F. \& Snyder, M. A single-molecule long-read survey of the human transcriptome. Nat. Biotechnol. 31, 1009-1014 (2013).

87. Tilgner, H., Grubert, F., Sharon, D. \& Snyder, M. P. Defining a personal, allelespecific, and single-molecule long-read transcriptome. Proc. Natl Acad. Sci. U. S.A. 111, 9869-9874 (2014).

88. Frankish, A. et al. GENCODE reference annotation for the human and mouse genomes. Nucleic Acids Res. 47, 767 (2019).

89. Harrow, J. et al. GENCODE: the reference human genome annotation for the ENCODE project. Genome Res. 22, 1760-1774 (2012).

90. Yates, A. D. et al. Ensembl 2020. Nucleic Acids Res. 48, D682-D688 (2020).

91. Yue, F. et al. A comparative encyclopedia of DNA elements in the mouse genome. Nature 515, 355-364 (2014)

92. Forrest, A. R. R. et al. A promoter-level mammalian expression atlas. Nature 507, 462-470 (2014).

93. Joglekar, A. A spatially resolved brain region- and cell type-specific isoform atlas of the postnatal mouse brain. https://doi.org/10.5281/ZENODO.4278162 (2020).

\section{Acknowledgements}

We thank Jenny Xiang, Dong Xu, and Adrian Tan at the Genomics Resources Core facility at Weill Cornell Medicine for help with facilitating sequencing, and Ishaan Gupta for helping with initial processing of the samples. We also thank Christopher Mason and Daniel Butler for use of their PromethION machine. This work was supported by the Brain Initiative (grant 1RF1MH121267-01 to H.U.T), NIGMS (grant 1R01GM135247-01 to H.U.T), NINDS (grant 1R01NS105477 to M.E.R), NIDA (T32DA03980 to S.L.), and NIMH (grant R01MH118934 to G.P.), Australian NHMRC Early Career Fellowship (APP1156531 to S.A.H), RFBR (grant 19-04-01074 to A.P.), D.R. was supported by Programma per Giovani Ricercatori Rita Levi Montalcini granted by the Italian Ministry of Education, University, and Research, by the Chan Zuckerberg Initiative DAF, an advised fund of Silicon Valley Community Foundation (CZF2019-002443), and by the NCI (2U24CA180996), S.A.S was supported by the Brain and Behavior Foundation NARSAD YIA and Sontag Foundation, P.F. was supported by NHGRI (grant 2U41HG007234), Wellcome (WT108749/Z/15/Z), and the European Molecular Biology Laboratory. E.D.J was supported by HHMI

\section{Author contributions}

A.J. and H.U.T. devised the experiments. P.G.C., S.L., A.K.S., B.H., O.F., M.Y.W., W.L., J.M., J.G.C., A.H., and N.I.W. performed the experiments. A.J., A.M., A.P., D.R., S.R.W. A.F., and H.U.T devised the analyses. A.J., A.P., A.M., Q.W., T.H., S.A.H., A.S., and H.U.T. performed the analyses. All of the authors discussed and interpreted the results throughout the project. A.J. and H.U.T. wrote the paper with inputs from all of the other authors. C.D., Z.B., D.R., E.D.J., O.F., S.A.S., A.F., P.F., W.L., G.S.P., A.B.S, M.E.R., and H.U.T. supervised the project.

\section{Competing interests}

J.G.C., A.H., N.I.W., S.R.W., and Z.B. are employees of 10X Genomics. PF is a member of the scientific advisory boards of Fabric Genomics, Inc., and Eagle Genomics, Ltd. A.J., A.P., A.M., P.G.C., S.L., A.K.S., J.M., B.H., M.Y.W., A.N.S., S.A.H., T.H., Q.W., C.D., O.F., S.A.S., D.R., E.D.J., W.L., G.P., A.F., A.B.S., M.E.R., and H.U.T. declare no competing interests.

\section{Additional information}

Supplementary information is available for this paper at https://doi.org/10.1038/s41467020-20343-5.

Correspondence and requests for materials should be addressed to H.U.T.

Peer review information Nature Communications thanks Mats Nilsson, Simone Codeluppi and the other anonymous reviewer(s) for their contribution to the peer review of this work. Peer reviewer reports are available.

Reprints and permission information is available at http://www.nature.com/reprints

Publisher's note Springer Nature remains neutral with regard to jurisdictional claims in published maps and institutional affiliations.

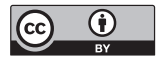

Open Access This article is licensed under a Creative Commons Attribution 4.0 International License, which permits use, sharing, adaptation, distribution and reproduction in any medium or format, as long as you give appropriate credit to the original author(s) and the source, provide a link to the Creative Commons license, and indicate if changes were made. The images or other third party material in this article are included in the article's Creative Commons license, unless indicated otherwise in a credit line to the material. If material is not included in the article's Creative Commons license and your intended use is not permitted by statutory regulation or exceeds the permitted use, you will need to obtain permission directly from the copyright holder. To view a copy of this license, visit http://creativecommons.org/ licenses/by/4.0/

(C) The Author(s) 2021 